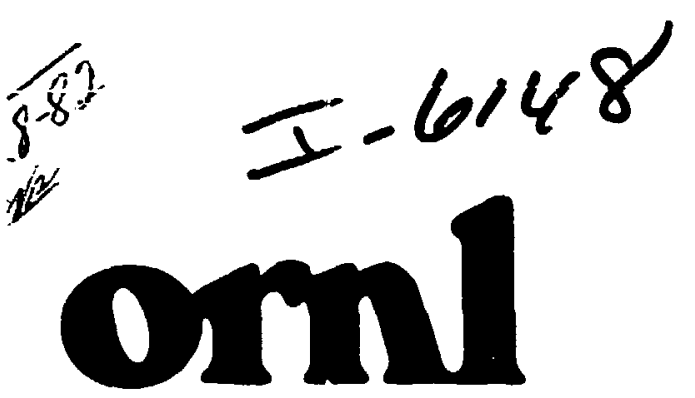

(2)

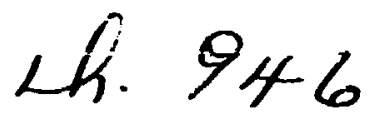

OAK

RIDGE

ORNL -5791

NATIONAL

LABORATORY

DE83 001719

\title{
Experimental and Calculational Analyses of Actinide Samples Irradiated in EBR-11
}

D. Gilai

M. L. Williams

J. H. Cooper

W. R. Laing

R. L. Walker

S. Raman

P. H. Stelson

UPERATED BY

UNION CARBIOE COBPORATION GOR THE UNTTED STATES UFPARTMENT OF ENEAGY 
Printed in the IInited States of America. Available from National Technical Information Service

U.S Department of Commerce

5285 Port Royal Road. Springfield, Virginia 22161

NTIS price codes-P-inted COpy: A05: Microfiche A01

This repon was prepared as an account of work sponsored by an agenc/ of the United States Government. Neither the United Stales Goverr.ment nor any agency thereot, nor any of ineir employeus, makes any warranty. express or implied. or assumes any legal liability or responsibility for the acruracy. complateriess. or usefuiness of any information. apparatus. procuct. or process disclosed. or represents inat its use would not infringe privately owned rights. Aeference herein to any specific commer wal product, process. or service by Irade name. irademark. manufacturev, or otherwise, does not nesessarily constitute or imply its endorsement. recommendation, or favoring by the United States Government or an's agency theieo;. The views and opinions of authors expressed herein do not iecessai ily state of reflect those ol the United States Government or any agency thereot. 


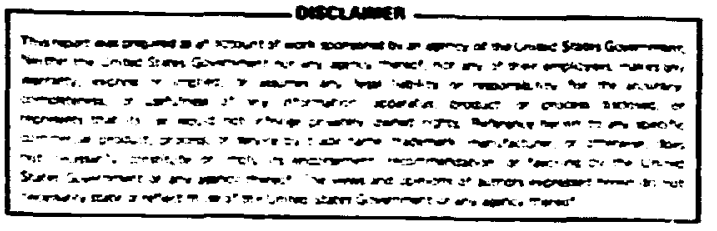

Contract fio. H-7405-eng-26
ORNL-5791

Dist. Category

UC-79d

\section{EXPERIMENTAL AND CALCULATIONAL ANALYSES}

OF ACTINIDE SAMPLES IRRADIATED

IN EBR-II

D. Gilai*

M. L. Millians

J. H. Cooper

H. R. Laing

R. L. Halker

S. Raman

P. H. Stelson
Engineering Physics Division

Analytical Chemistry Division

Physics Division

Date Published - October 1982

This Nork was Sponsored by

U.S. Department of Energy

Division of Basic Energy Sciences

*Nuclear Research Ceniter Negev, ?.0. Box 9001, Beer-Sheva, ISRAEL

OAK RINGE NATIONAL LABORATURY

Oak Rfdge, Tennessee 37830

operated by

UNION CARBIDE CORPORAIION

for the

CEPARTMENT OF ENERGY 
TABLE OF CONTENTS

ABSTRACT $\ldots \ldots \ldots \ldots \ldots \ldots \ldots \ldots \ldots \ldots \ldots \ldots \ldots \ldots \ldots \ldots \ldots \ldots \ldots \ldots \ldots \ldots \ldots \ldots \ldots$

ACKNOULEDGRENTS $\ldots \ldots \ldots \ldots \ldots \ldots \ldots \ldots \ldots \ldots \ldots \ldots \ldots \ldots \ldots \ldots \ldots \ldots \ldots \ldots$

I. INTRODUCTION $\ldots \ldots \ldots \ldots \ldots \ldots \ldots \ldots \ldots \ldots \ldots \ldots \ldots \ldots \ldots \ldots \ldots \ldots \ldots \ldots$ I

I1. DESCRIPTION OF THE EXPERIMENTAL ENVIRONMENT $\ldots \ldots \ldots \ldots \ldots \ldots \ldots \ldots 5$

III. THE EXPERIMENTAL DETERMIMATION OF THE SAMPLES COMPOSITION ... 9

IV. THE CALCULATIONAL AMALYSIS OF IRRADIATED SAMPLES ......... 17

V. COMPARISON OF CALCULATIONAL AND EXPERIMENTAL RESULTS ....... 37

VI. SENSITIVITY ANALYSIS OF INPUT PARAMETERS AND THEIR IMPACT ON

CALCULATED RESPONSES .............................. 55

VII. SUMLARY AMD COHCLLISIONS $\ldots \ldots \ldots \ldots \ldots \ldots \ldots \ldots \ldots \ldots \ldots \ldots \ldots \ldots \ldots . \ldots 1$

VIII. REFERENCES $\ldots \ldots \ldots \ldots \ldots \ldots \ldots \ldots \ldots \ldots \ldots \ldots \ldots \ldots \ldots \ldots \ldots \ldots \ldots \ldots \ldots$

APPENDIX A. IRRADIATION HISTORY OF THE ACTINIDE SAMPLES IN EBR-II * 79

APPENDIX B. PROCEDURE FOR THE DISSOLUTION OF EBR-II IRRADIATED

NICKEL CAPSULES CONTAINING PU02 ................ 83 
ABSTRACT

Higher actinides influence the characteristics of spent and recycled fuel and dominate the long-tern hazards of the reactor waste. Reactor irradiation experiments provide useful benchmarks for testing the evaluated nuclear data for these actinides. During 1967-1970, several actinide samples were irradiated in the Idaho FBR-II fast reactor. These samples have now been analyzed, employing mass and alpha spectometry, to determine the heavy element products. A simple spherical model for the EBR-II core and a recent version of the ORIGEN code with ENDF/B-Y data were employed to calculate the exposure products. A detailed comparison between the experimental and calculated results has been made. For samples irradiated at locations near the core center, agreement within $10 \%$ was obtained for the major isotopes and their first daughters, and within $20 \%$ for the nuclides up the chain. A sensitivity analysis showed that the assumec flux should be increased by $10 \%$. The lessons learned from the present study were incorporated into the planning of a future irradiation study to be carried out in the UK Prototype Fast Reactor at Dounreay. 


\section{ACKMOULEDGEMENTS}

The experiment involving irradiation of actinide samples in EBR-II was initiated by the scientific personnel at the Argonne Mational Laboratory. Their primary objective was to determine accurately the fission yields of various pctential burnup monitors for application to fast reactors. A secondary objective was the study of heavy element transformations of the actinides. Beyond accomplishing the irradiations and the analysis of a single ${ }^{239} \mathrm{Pu}$ sanple, this experiment was disr.ontinued, for various reasons, at AN'. When requested, the irradiated samples were readily made available to us along with bickground information. He acknowledge deeply the assistance received from $R$. R. Heinrich, J. J. Hines, R. P. Larsen, D. Meneghetti, R. J. Meyer, R. D. 0ldham, R. J. Popek and j. P. Unik, all from ANL. Some samples were shared with H. J. Maeck at Idaho. He thank J. E. Bigelow, J. A. Carter, J. H. T. Dabbs, M. C. G. Hall, C. R. Weisbin and J. R. White, all fram ORIL, for their interest in this work and for several helpful comments and criticisims. He also thank S. Whetstone of the U.S. Dapartment of Energy for his interest and encouragement. 


\section{INTRODUCTION}

In performing fast reactor fuel cycle analysis, burnup calculations are done to determine not only the time-dependent reactor operating conditions and performance, but also many factors wich may influence spent fuel reprocessing/fasrication and waste disposal. Calculations of reactor core performance (e.g., reactivity swing, breeding, etc.) depends largely on the accuracy of the cross sections for the "cormon" uranium and plutonium isotopes $\left({ }^{238} \mathrm{U},{ }^{235} \mathrm{U} ;{ }^{239} \mathrm{Pu},{ }^{240} \mathrm{Pu},{ }^{241} \mathrm{Pu}\right)$. However, many of the factors which affect the ex-core portion of the fuel cycle depend on knowledge of the buildup of less common nuclides, such as ${ }^{238} \mathrm{Pu}$ and the $\mathrm{Am}$ and $\mathrm{Cm}$ isotope!. These nuclides influence the chararteristics of the spent and recycled fuel through their contributions to neutron heating and source strength and dominate the long-term hazard of the reactor waste. The calculated production of these nucitides depends on the more "eyotic" nuclear data of the trans-plutonium actinides, which until recent: $y$ has been poorly represented on the ENOF/B data files. The latest release of ENDF/B, version $V$, supposedly has much improved data for the higher actinides; however, the new data have never actually been tested against actual fast reactor experiments.

Reactor irradiation experiments an provide an iritegral comparison of measurement versus calculation and hence are useful as benchmarhs for data testing. While this type of information is avallable for several thermal reactor cores, very little experimental information exists on the transmutation of actinides irradiated in fast reactors. Until the advent of the Fast flux Test Factlity (FFTF), the Experimental Breeder 
Reactor (EBR-II) was the only fast reactor available in the U.S. for such experiments, and its spectrum is somewhat harder than the spectrum in proposed commercial LMFBR's. A joint U.S. and United Kingdom program is currently under way to irradiate many actinide samples in the English Prototype Fast Reactor (PFR). The actinide samples are to be provided by the U.S. and will include such nuclides as $231 \mathrm{~Pa}, 241 \mathrm{Am},{ }^{243} \mathrm{Am},{ }^{243} \mathrm{~cm}$, ${ }^{244} \mathrm{~cm}$, and ${ }^{245} \mathrm{~cm}$, as well as the more common thorium, uranium, and plutonium isotopes. Pure samples of these isotopes are very valuable and therefore prior to the proposed PFk irradiation, similar irradiation experiment done in EBR-II has bee, nalyzed to obtain experience and to reveal any pitfalls in performing fast reactor experiments, which hopefully could be avoided in the PFR experiment.

During the years 1967-1970 several actinide samples ranging from 232 Th to $: 1 \mathrm{Pu}$ were irradiated in the Idaho Experiment, 1 Breeder Reactor EBR-11. The actinides examined in this experiment, and their exposure products, are of importance for physical analysis and economical evaluation of the in-core portion of fast breeder reactor fuel cycles. The original goal of our efforts was to test methods and nuclear data used in fast reactor core calculations; however, the goal was extended to also provide recommended procedures for the future PFR experiment. The purpose of this report therefore is to present results of, as well as to review critically, the calculational and experimental procedures used to determine the exposure products in the irradiated samples. 
The burnup or buildup of actinides in a sample may be derived from the difference between the sample's isotopic compositions before and after irradiation, the former being measured not directly for the sample itself but for an identical unirradiated control sample. Analyses of one of the irradiated (as well as the unirradiated) EBR samples began soon after they had been discharged from the reactor, by the Chemistry Division at ANL: however, all other samples remained untouched until 1978 when ORNL startea a program to complete the analysij. This work was finished in early 1981.

In the next chapter a description of the experimental environment as it concerns the present study is given. A description of the experimental techniques used for sample analysis and a detailed description of the calculational model are given in Chapters III and IV respectively. The experimental and calculational results are compared in chapter $V$. This chapter also discusses the adequacy of the ca!culational procedures employed in the study. Since discrepancies between calculation and experiment were found for some samples, a series of sensitivity calculations were performed in order to reveal possible sources of errors. The results of these calculations are given in Chapter VI. Concluding remarks and recommendations for future experiments are given in Chapter VII. 


$$
4
$$


II. DESCRIPTION OF THE EXPFPIMFNTAL ENVIRONMENT

The EBR-II reactor configiration at the time of this experiment consisted of a driver core n: highly enriched ( 5,0\%) uranium, surrounded by a dep!ated $238 \mathrm{U}$.blanket. The ruel was arranged in a hexagonal array of 91 subassemblies of wich 16 locations wire reserved for experimental purposes. I The lattice pitch was $5.89 \mathrm{~cm}$ and the core iright varied between 34.3 and $36.1 \mathrm{~cm}$. The experimental actinide samples were stacked in two sealed tubes. The first tube, containing 12 samples, was placed in the driver core and the second, containing 8 samples, was placed below the firs: one in the axial blunket. The ordering of the samples in the tutes is given in figure 1.

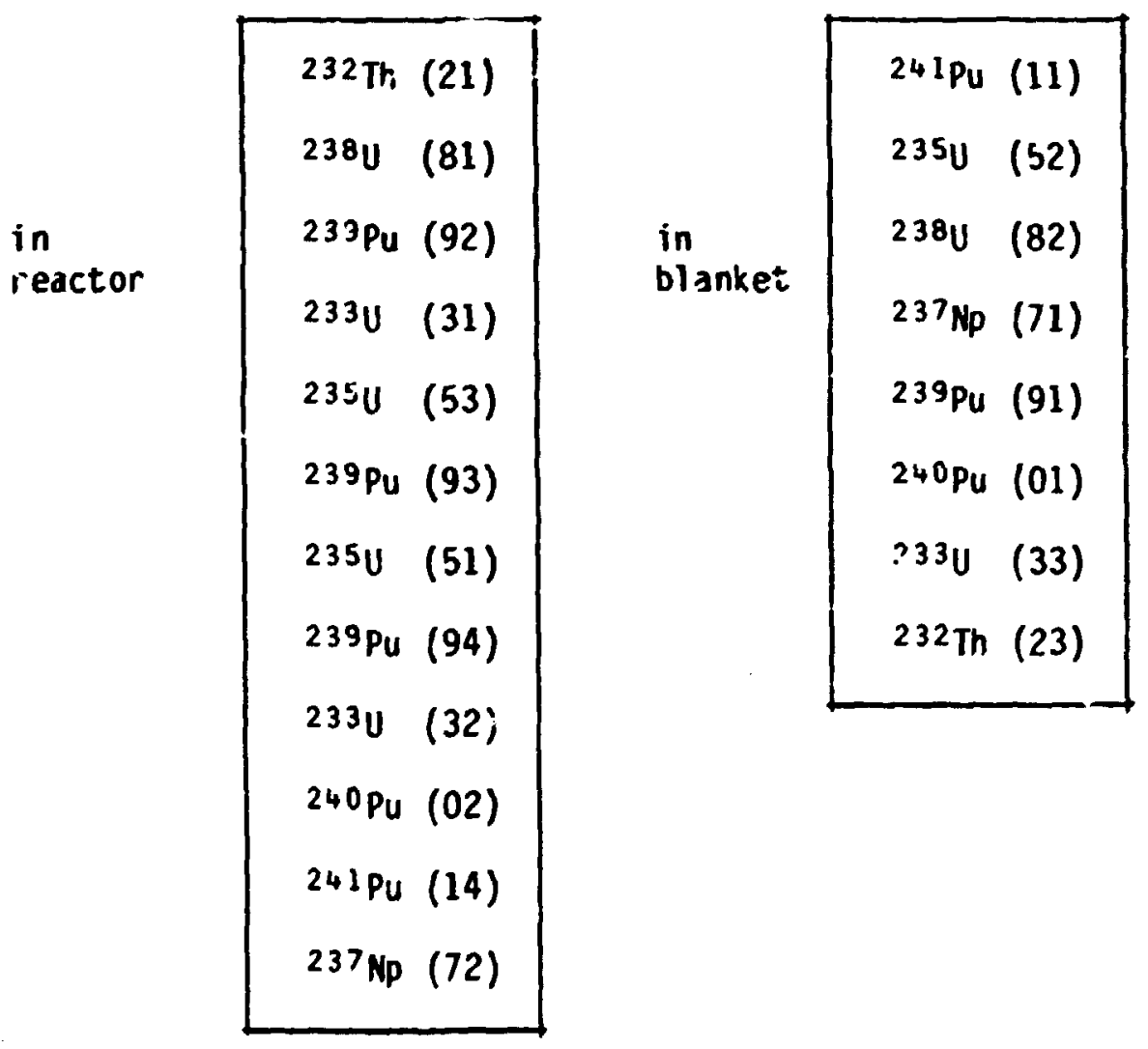

Figure 1. Sampies Placement in EBR-II 
Each of the 20 samples had an identity number shown in Figure 1. These numbers were given at the early stage of the experiment and are used in the present report for compatibility with other reports.

The sample materials, usually about $100 \mathrm{mg}$ in weight, were lonsely packef oxide powders sealed in cylindrical nickel containers. 2 The outer diameter of each container was $0.912 \mathrm{~cm}$ and their wall thickriess was $0.185 \mathrm{~cm}$. Each cylinder was $2.87 \mathrm{~cm}$ high so that the total length of 12 stacked containers was equivalent to a core height of $34.3 \mathrm{~cm}$. To protect against hazards which might be caused by a failure of the containers, the reactor and the blanket samples were separately assembled into two sealed nickel capsules, wich were designed to keep fission products from entering the coolant. These capsules also contained small amounts of NaK for better heat removal.

nue to its nature as an experimental reactor, the EB?-II core was continously changing during the irradiation period. These changes should be taken into consideration since they could affect the burnup history of the samples by causing flux levels and spect,ra to vary from loading to ioading. However, it was cbserved ${ }^{3}$ that the relative fluence distribution along a core radius remained almost constant from run to run from which it was inferrod that the relative flux distribution also remained nearly constant. Another observation was that the measured values of $\left(\sigma \mathrm{c} / \sigma_{f}\right)$ for many isotopes do not change during many EBR runs, ${ }^{4}$ which leads to the conclusion that the flux spectrum is nearly constant t,o0. Therefore it has been assumed that a single representative model of the reactor at a characteristic time 
will be sufficient for all calculations; i.e., the only time-dependent variable which has to be considered is the power level of the reactor. This parameter was provided by ANL and was used in the depletion calculations described later. The majority of this information may be found in Ref. 5. 
$\$$ 


\section{THE EXPERIMENTAL DETERMINATION OF THE SAMPLES COMPOSITION}

\section{A. INTRODUCTION}

Mass spectrometry has been used to measure the isotopic distribution and total quan ty cf uranium and plutonium in irradiated samples from the EBR-II reactor. Tracers of U-233, U-236, and PU..242 were used for quantitative analysis ty isotope dilution. Neptunium and actinide products formed by nuclear reactions were measurest by alpha spectrometry. Burn-up calculations have been made to compare with the results obtained from these measurements.

\section{B. EXPERIMENTAL}

\section{i. INSTRUMENTAL}

The mass sp-ctrometer measurements were made using a 2-stage, 30-cm radius thermal emission mass spectrometer of ORNL design. ${ }^{6}$ High vacuum is achieved by ion pumping, with normal operating pressures $<4 \times 10^{-5} \mathrm{~Pa}$. A pulse-counting detection system, comprised of an RCA 6812 iectron multiplier as the detector, an ORTEC discriminator, an ORTEC scaler, and an ARL preamplifier, is used for higher sensitivity. This system, which operates under the control of a Digital Equipment Corporation PDP-11/10 computer, scans the mass range by adjusting the high voltage source. Only peak tops are scanned, and it is possible to sweep each peak top a preselectable number of times for each scan across the mass region. For the analysis of plutonium and uranium, a background correction is made to all masses. 
Measurements made on nuciides such as Kp-237, Pu-239, and Pa-231 were done using gas-flow, alpha counters and pulse height anclysis systems conmon to radiochemical laboratories.

\section{SAMPLE DISSOLUTION}

The first step in the analysis of irradiated targets is to prepere a clean area for sample dissolution. A hot cell was cleared of all unneeced zquipment and cleaned thoroughly. Both detergents ana acid washes were used with remote scrubbing and pressure washers.

New quartz reflux flasks and water-cooled condensers were placed in the cell. Each compact was weighed using an analytical balance with $0.1 \mathrm{mg}$ sensitivity and placed in the quartz flask. Nitric acid was used to dissolve the targets according to the procedure in Appendix B. An addition of a small amount of hydrufluoric acid aided the dissolution of samples containing thorium or plutonium. After dissoiution was complete (4-24 hours) the solution was transferred to a new voiumetric flask, made co volume, and weighed to $\pm 0.1 \mathrm{mg}$. Aliquots were removed from this solution for the measurement of individual elements or isotopes. Duplicate blanks were carried through the entire process with each set of samples.

\section{SAMPLE PREPARATION}

A weight basis was employed for all aliquanting and dilutions to improve the precision and accuracy of the analyses. A simplified resin bead sample preparation procedure for plutonium and uranium isotopic composition has been developed $a^{t}, \mathrm{ORNL}^{7}$ and used for this work. In this method, several anion resin beads are introduced into a solution of the sample in $8 \mathrm{M} \mathrm{HNO}_{3}$ to absorb $\mathrm{Pu}, \mathrm{J}$, or Th. A single bead 
i; removed and transferrec to a rheniun V-shaped filament for mass analysis. The amount of each element on a single bead is severa? nanograms, which is adequate for a sequential analysis of plutoniun ard uranium or thorium. For this work, only zone-refined rhinium was used for making filaments, and they were cleaned after manufacture by baling at $2000 \mathrm{C}$ for 30 minutes.

Where alpha counting was this choice for quantitative analysis, solvent extraction techniques were employed to prepare purified sanpies. Methylisobutyl ketone was used to extract $\mathrm{Np-237}$ and Pu-238 and dijscbutyl carbinol was used for extraction of $P_{\dot{a}}-231$.

\section{RESULTS}

The primary target isotopes used in this experiment were $U-233$, U..235, U-238, Pu-239, Pu-čco, and Pu-241. The results for the initiai, unirradiated isotopic compositions are given in Tahle 3.1. Isotopic data for the starting fuel were taken from an ANL report ${ }^{2}$ and confirmed by recent ORKL measurements made on archive samples. There is very good agreement between the two sets of data. No correction for decay of Pu isotopes was made on ORNL data; however, if one uses 16 years since ANL analysis for this calculation, plutonium isotopic results agree well. For this experiment, this approximation was sufficient for confirmation.

The results for the primary target nuclides are given in Tables 3.2 and 3.3. The quantity of fuel loaded into targets and the quantity measured by tsotope dilution after irradiation ara shown. Precisions quoted for all isotopic measurements are given under the individual 
Table 3.1 Unirradiated Iraniun and Flutonium Isotupic Compositions

\begin{tabular}{|c|c|c|c|}
\hline $\begin{array}{c}\text { Major } \\
\text { I sotope }\end{array}$ & Isetope & $\begin{array}{c}\text { Composition }(x) \\
\text { NLI }^{a}\end{array}$ & $\begin{array}{l}\text { Composition }(x) \\
\text { ORNLE } \pm \text { S.O. }\end{array}$ \\
\hline$U-233$ & $\begin{array}{l}233 \\
234 \\
235 \\
236 \\
238\end{array}$ & $\begin{array}{c}98.51 \\
1.06 \\
0.012 \\
- \\
0.414\end{array}$ & $\begin{aligned} 98.518 & \pm 0.003 \\
1.051 & \pm 0.003 \\
0.019 & \pm 0.001 \\
<0.001 & \\
0.412 & \pm 0.006\end{aligned}$ \\
\hline$U-235$ & $\begin{array}{l}234 \\
235 \\
236 \\
238\end{array}$ & $\begin{array}{c}0.90 \\
93.16 \\
0.438 \\
5.50\end{array}$ & $\begin{array}{r}0.908 \pm 0.001 \\
93.250 \pm 0.015 \\
0.436 \pm 0.002 \\
5.406 \pm 0.013\end{array}$ \\
\hline$U-238$ & $\begin{array}{l}234 \\
235 \\
236 \\
238\end{array}$ & $\begin{array}{c}- \\
0.037 \\
- \\
99.96\end{array}$ & $\begin{array}{c}0.0010 \pm 0.0001 \\
0.045 \pm 0.001 \\
0.0018 \pm 0.0002 \\
99.952 \pm 0.001\end{array}$ \\
\hline$P y-239 C$ & $\begin{array}{l}239 \\
240 \\
241 \\
242\end{array}$ & $\begin{array}{l}95.17 \\
4.59 \\
0.218 \\
0.019\end{array}$ & $\begin{array}{l}\text { No archive sample } \\
\text { available. }\end{array}$ \\
\hline$P u-240$ & $\begin{array}{l}238 \\
239 \\
240 \\
241 \\
242\end{array}$ & $\begin{array}{r}0.77 \\
98.58 \\
0.54 \\
0.11\end{array}$ & $\begin{aligned} & C .0024 \pm 0.0001 \\
& 0.825 \pm 0.004 \\
& 98.798: 0.005 \\
& 0.2=3 \pm 0.002 \\
& 0.121 \pm 0.001\end{aligned}$ \\
\hline$P u-241$ & $\begin{array}{l}238 \\
239 \\
240 \\
241 \\
242\end{array}$ & $\begin{array}{r}- \\
1.03 \\
3.66 \\
94.04 \\
1.27\end{array}$ & $\begin{aligned} 0.0152 & \pm 0.0002 \\
2.094 & \pm 0.009 \\
7.539 & \pm 0.009 \\
87.853 & \pm 0.009 \\
2.499 & \pm 0.6502\end{aligned}$ \\
\hline
\end{tabular}


Table 3.2 Isotopic Composition of Uranium in Irradiated Samples

\begin{tabular}{|c|c|c|c|c|c|c|c|}
\hline \multirow{2}{*}{$\begin{array}{l}\text { Majord } \\
\text { Isotope }\end{array}$} & \multicolumn{2}{|c|}{ wt. of $U_{2} \mathrm{mg}$} & \multicolumn{5}{|c|}{ Isotope Composition of $U(x)$} \\
\hline & $\begin{array}{l}\text { Before } \\
\text { Irradiation }\end{array}$ & $\begin{array}{l}\text { After } \\
\text { Irradiation }\end{array}$ & 233 & 234 & 235 & 236 & 238 \\
\hline$U-233\{31\}$ & 100.07 & $\begin{array}{l}82.74 \\
\pm 0.13\end{array}$ & $\begin{array}{l}96.827 \\
\pm 0.006\end{array}$ & $\begin{array}{r}2.552 \\
\pm 0.002\end{array}$ & $\begin{array}{r}0.076 \\
\pm 0001\end{array}$ & $\begin{array}{c}0.0014 \\
\pm 0.001\end{array}$ & $\begin{array}{r}0.544 \\
\pm 0 . \wedge 04\end{array}$ \\
\hline U233(32) & 134.48 & $\begin{array}{r}113.4 \\
\pm 2.0\end{array}$ & $\begin{array}{r}.966 \\
\pm 0.01\end{array}$ & $\begin{array}{c}2.50 \\
\pm 0.001\end{array}$ & $\begin{array}{r}0.060 \\
\pm 0.001\end{array}$ & $\begin{array}{r}0.007 \\
\pm 0.001\end{array}$ & $\begin{array}{r}0.47 \\
\pm 0.01\end{array}$ \\
\hline$U-233(33)$ & 57.81 & $\begin{array}{l}48.76 \\
\pm 0.24\end{array}$ & $\begin{array}{l}96.222 \\
\pm 0.022\end{array}$ & $\begin{array}{r}3.080 \\
\pm 0.022\end{array}$ & $\begin{array}{r}0.194 \\
\pm 0.004\end{array}$ & $\begin{array}{r}0.005 \\
\pm 0.001\end{array}$ & $\begin{array}{r}0.500 \\
\pm 0.014\end{array}$ \\
\hline$U-235(53)^{b}$ & 88.19 & $\begin{array}{l}79.3 \\
\pm 0.5\end{array}$ & - & $\begin{array}{r}0.89 \\
\pm 0.02\end{array}$ & $\begin{array}{l}90.51 \\
\pm 0.02\end{array}$ & $\begin{array}{r}2.69 \\
\pm 0.01\end{array}$ & $\begin{array}{r}5.92 \\
\pm 0.02\end{array}$ \\
\hline$U-238(81)^{c}$ & 91.58 & $\begin{array}{l}90.30 \\
\pm 0.4\end{array}$ & - & $\begin{array}{r}0.000 ? \\
\pm 0.0002\end{array}$ & $\begin{array}{r}0.035 \\
\leq 0.001\end{array}$ & $\begin{array}{r}0.0014 \\
\pm .0002\end{array}$ & $\begin{array}{l}99.963 \\
\pm 0.001\end{array}$ \\
\hline$U-238(82)$ & 87.85 & $\begin{array}{l}84.07 \\
\pm 0.42\end{array}$ & - & $\begin{array}{r}0.0018 \\
\pm 0.0002\end{array}$ & $\begin{array}{r}0.033 \\
\pm 0.001\end{array}$ & $\begin{array}{r}0.0015 \\
\pm 0.0002\end{array}$ & $\begin{array}{l}99.963 \\
\pm 0.001\end{array}$ \\
\hline$T h-232(21)^{d}$ & 0.00 & $\begin{array}{r}0.58 \\
\pm 0.04\end{array}$ & $\begin{array}{l}98.60 \\
\pm 0.1\end{array}$ & $\begin{aligned} & 0.90 \\
& \pm 0.1\end{aligned}$ & $\begin{array}{r}0.17 \\
\pm 0.03\end{array}$ & - & $\begin{array}{r}0.42 \\
\pm 0.05\end{array}$ \\
\hline
\end{tabular}

a Capsule number in parentheses.

bMore uncertainty surrounding these data. Sample was dissolved at ANL or ldaho Falls; solutions sent to ORIL for analysis. All others

received in capsules and dissolved at ORNL.

CPu composition for this sample is listed in Table 5.4.

dinitial weight of Th before irradiation was $32.81 \mathrm{mg}$. 
Table 3.3 Isotopic Composition of Plutontum In Irradiated Samples

\begin{tabular}{|c|c|c|c|c|c|c|c|}
\hline \multirow[b]{2}{*}{$\begin{array}{l}\text { Majora } \\
\text { I sotope }\end{array}$} & \multicolumn{2}{|c|}{ Wt. of $\mathrm{Pu}, \mathrm{mg}$} & \multicolumn{5}{|c|}{ Isotopic Composition of Pu (x) } \\
\hline & $\begin{array}{l}\text { Before } \\
\text { Irradiation }\end{array}$ & $\begin{array}{l}\text { After } \\
\text { Irradiation }\end{array}$ & 238 & 239 & 240 & 241 & 242 \\
\hline $\mathrm{Np}-237(72) \mathrm{C}$ & 0.0 & $\begin{array}{l}.056 \\
\pm .0017\end{array}$ & 100.00 & & & & \\
\hline$P u-239(94)$ & 105.74 & $\begin{array}{l}89.93 \\
\pm 1.00\end{array}$ & $\begin{array}{r}0.016 \\
\pm 0.002\end{array}$ & $\begin{array}{l}93.247 \\
\pm 0.016\end{array}$ & $\begin{array}{r}6.476 \\
\pm 0.016\end{array}$ & $\begin{array}{r}0.234 \\
\pm 0.003\end{array}$ & $\begin{array}{r}0.026 \\
\pm 0.001\end{array}$ \\
\hline $\mathrm{Pu}-239(91)$ & 71.35 & $\begin{array}{l}66.56 \\
\pm 0.34\end{array}$ & $\begin{array}{r}0.022 \\
\pm 0.001\end{array}$ & $\begin{array}{l}90.588 \\
\pm 0.002\end{array}$ & $\begin{array}{r}8.771 \\
\pm 0.002\end{array}$ & $\begin{array}{r}0.577 \\
\pm 0.003\end{array}$ & $\begin{array}{r}0.041 \\
\pm 0.001\end{array}$ \\
\hline$P u-239(92)^{b}$ & 82.99 & $\begin{array}{l}82.00 \\
\pm 0.2\end{array}$ & $\begin{array}{r}0.019 \\
\pm 0.002\end{array}$ & $\begin{array}{l}93.148 \\
\pm 0.015\end{array}$ & $\begin{array}{r}6.555 \\
\pm 0.015\end{array}$ & $\begin{array}{r}0.251 \\
\pm 0.002\end{array}$ & $\begin{array}{r}0.026 \\
\pm 0.001\end{array}$ \\
\hline$P u-240(02)$ & 51.44 & $\begin{array}{l}47.54 \\
\pm ? .12\end{array}$ & $\begin{array}{r}0.012 \\
\pm 0.001\end{array}$ & $\begin{array}{r}0.753 \\
\pm 0.004\end{array}$ & $\begin{array}{l}97.768 \\
\pm 0.004\end{array}$ & $\begin{array}{r}1.324 \\
\pm 0.005\end{array}$ & $\begin{array}{r}0.143 \\
\pm 0.002\end{array}$ \\
\hline$P u-241(14)^{b}$ & 32.84 & $\begin{array}{l}15.64 \\
\pm 0.20\end{array}$ & $\begin{array}{r}1.94 \\
\pm 0.01\end{array}$ & $\begin{array}{r}2.00 \\
\pm 0.01\end{array}$ & $\begin{array}{r}7.25 \\
\pm 0.05\end{array}$ & $\begin{array}{l}83.78 \\
\pm 0.05\end{array}$ & $\begin{array}{r}5.03 \\
\pm 0.04\end{array}$ \\
\hline$P u-241(11)$ & 26.31 & $\begin{array}{l}10.13 \\
\pm 0.02\end{array}$ & $\begin{array}{r}3.245 \\
\pm 0.008\end{array}$ & $\begin{array}{r}1.903 \\
\pm 0.015\end{array}$ & $\begin{array}{r}6.176 \\
\pm 0.024\end{array}$ & $\begin{array}{l}81.185 \\
\pm 0.025\end{array}$ & $\begin{array}{r}6.891 \\
\pm 0.015\end{array}$ \\
\hline
\end{tabular}

aCapsule number in parentheses.

byore uncertainty surrounding these data. Samples were dissolved at ANL

or Idaho Falls; solutions sent to ORNL for analysis. All others re-

ceived in capsules and dissolved at ORNL.

CInitial weight of $\mathrm{Np}$ before irradiation nas $1.0 \mathrm{mg}$. 
results; they are standard deviation values which represent internal precision. The accuracy of the measurements is expected to be better than one percent except in cases indicated by the footnote $b$ in Tables 3.1 and 3.2 . 
14 


\section{THE CALCULATIONAL AMALYSIS OF IRRADIATED SAMPLES}

\section{A. SEPARATION INTO SPACE- AND TIME-DEPERDEHT PROBLEMS}

A complete calculational analysis of the irradiation process requires a solution of the coupled neutron-nuclide equations in space, energy, and time. However, for long irradiation periods and frequent civanges in core composition, such an approach becomes ioo elaborate and expensive for this type of experiment. A frequently used approximation is to assume space-time separability by splitting the equations into two sets, one being time independent and the other being space independent. Such an approach is applicable for the analysis of EBR-II since as stated above, its flux distribution and spectrum (but not flux level) remained essentially constant during the who'e period of irradiation. The calculation of the exposure products at the end of irradiation was therefore performed in two steps. In the first step, the flux spatial shape ard spectrum were calculated for a representative EBR-II model. ${ }^{8}$ The flux spectrum was then used to collapse a multigroup cross section set into a one-group library. In the second step, the one-group cross sections, along with the flux level history of the EBR-II, were utilized in a series of depletion calculations which provided the amounts of exposure products in each sample. A detailed description of this approach will follow next.

B. THE SPACE-DEPENDENT PROBLEM: DETERMINATION OF FLUX SPECTRUM ANO ONE-FRROUP CROSS SECTIONS

During the perfod in which the actinide samples were frradiated in EBR-I1, the reactor was started up and shut down more than 60 times. 
In most of the shutdown periods, fresh fuel subassemblies were loaded or spent fuel subassemblies were discharged while some others were shuffled for experimental purposes. The core configuration was therefore continuously changing, so that it is difficult to determine a "typical" configuration out of the many different core loarings. Yot, since our purpose is to check the adequacy of a simple modsl for burilup analysis. we will focus the analysis on a model, which although containing few details about the fine structure of the core composition, may still be regarded as a representative model for many core lcadings. This model is described in Ref. 8 and is basically a onedimensional spherical reactor, divided in three different concentric zones: an inner driver core, a reflector, and a blanket. The material composition in each zone is assumed to be homogeneous so that there are no irregularities other than the differences between the three zones in the model. The effects of localized heterogeneities on the flux are usually small for fast reactors because of the long mean free path of high energy neutrons. The materials comprising each zone and their atom densities are listed in Table 4.1 .

In order to judge how adequate this simplified model is, and to justify several assumptions made later, a picture of the simplifien model overlapping the real reactor configuration is given in Fig. 2. This picture shows that the inner core of EBR-11 may hardly be described as a sphere becausc its diameter-to-height ratio is far away from 1.0. Since only the center zones of both models do overlap, it is difficult to locate the sample positions in the simplicied model, especially for those near the inner core boundary. 


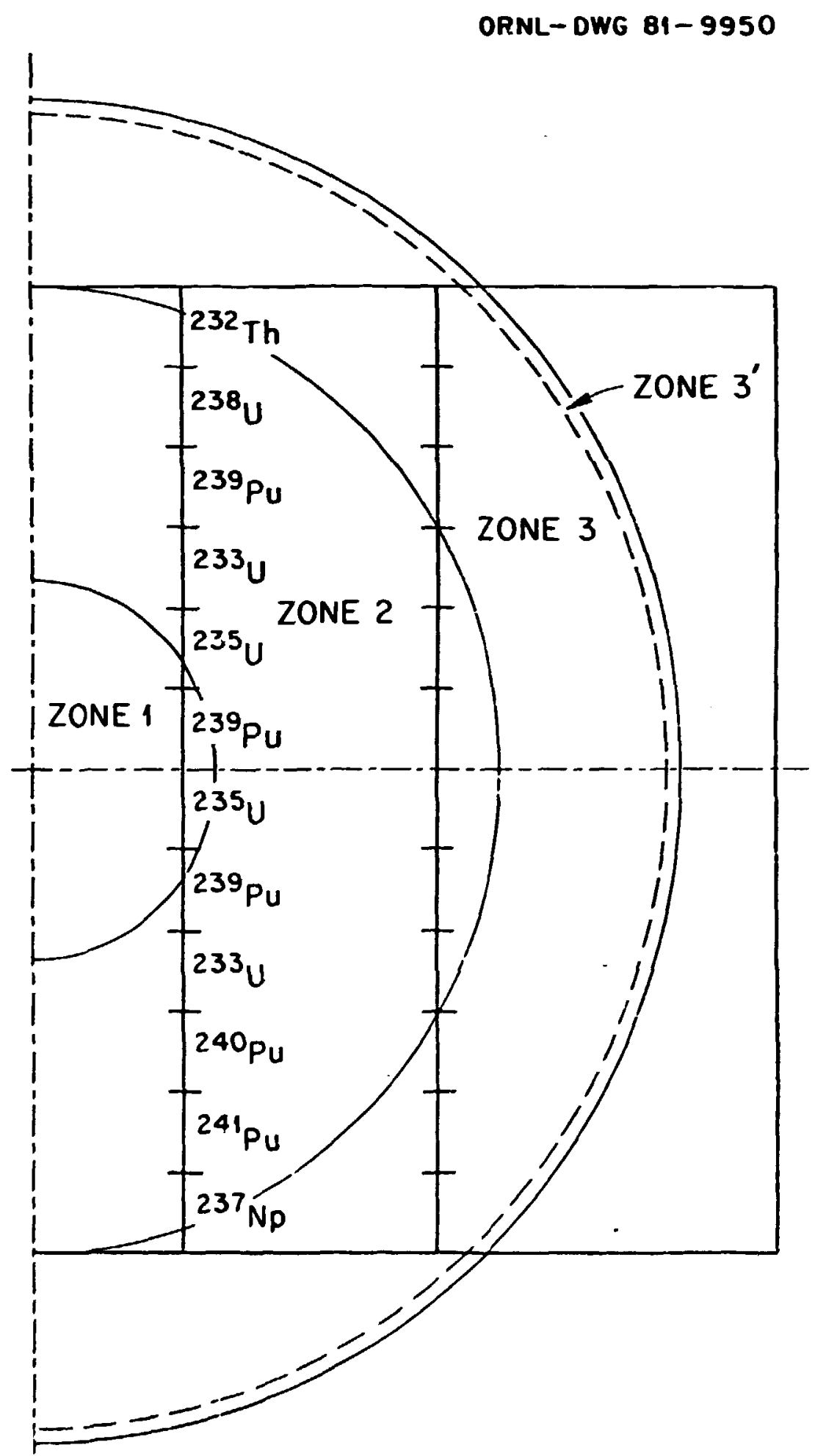

Fig. 2: The EBR-II sfilerical model compared to the real geometry of EBR-I I 
Table 4.1 Composition of simplified EBR-II Model

\begin{tabular}{lccc} 
& \multicolumn{3}{c}{ Concentration (atoms $/ \mathrm{cm}^{3 \star} 10^{24}$ ) } \\
\cline { 2 - 4 } Material & $\begin{array}{c}25.2 \mathrm{~cm} \\
\text { Radius Core }\end{array}$ & $\begin{array}{c}10 \mathrm{~cm} \text { thick } \\
\text { Reflector }\end{array}$ & $\begin{array}{c}35 \mathrm{~cm} \text { thick } \\
\text { Blanket }\end{array}$ \\
\hline $235 \mathrm{U}$ & $6.3 \cdot 10^{-3}$ & - & $6.0 \cdot 10^{-5}$ \\
$238 \mathrm{U}$ & $6.0 \cdot 10^{-3}$ & - & $2.9 \cdot 10^{-2}$ \\
$2{ }^{39 P u}$ & $1.0 \cdot 10^{-5}$ & - & $3.0 \cdot 10^{-5}$ \\
$\mathrm{Fe}$ & $1.2 \cdot 10^{-2}$ & $4.5 \cdot 10^{-2}$ & $1.2 \cdot 10^{-2}$ \\
$\mathrm{Ni}$ & $1.5 \cdot 10^{-3}$ & $6.1 \cdot 10^{-3}$ & $1.5 \cdot 10^{-3}$ \\
$\mathrm{Cr}$ & $3.0 \cdot 10^{-3}$ & $1.4 \cdot 10^{-2}$ & $3.0 \cdot 10^{-3}$ \\
$\mathrm{Na}$ & $1.1 \cdot 10^{-2}$ & $4.0 \cdot 10^{-3}$ & $4.0 \cdot 10^{-3}$ \\
$\mathrm{Mo}$ & $7.0 \cdot 10^{-4}$ & - & - \\
\hline
\end{tabular}

The exact location of the samples however, is for two reasons most important: firstly because the location of the sample determines the flux level to which its nuclides are exposed, and secondly because the flux spectrum varies from point to point. It is important to use the flux spectrum ertaining to the location of the sample in order to obtain appropriate?y collapsed cross sections for the subsequent depletion calculations. To overcome the above mentioned difficulty, spatial flux distributions wer taken from earlier two-aimensional calculations provided by ANL instead of from the simplified model calculations, which was used merely to obtain flux spectra for different sample locations. To that end the inner cure was divided into three subzones, each lepresenting a part of the core in which only small flux spectra variations are expected. The radif oi these subzones were chosen somewhat arbitrarily and are also shown in Figure 2. 
At the time the samples were first introduced into the reactor they were located at row 2, which is one lattice pitch off center. Later on (from run 34A) they were moved to row 4, where they remained until their final discharge fwith exception of short periods in which they were entirely withdrawn from the reactor). From figure 1 it can be seen that the change in the position of the samples subassembly causes each sample to move into the next subzone of the driver core, a fact wich has to be taken in consideration when choosing the appropriate spectrum-averaged cross sections for subsequent depletion calculations.

From EBR-II reports it can be learned that besides the changes in core loading from run to run, which may be regarded as minor changes, several major changes also took place. The most important change is the transition from MARK-i to MARK-1A type driver fuel. During this time the reactor axial blanket was also changed from depleted uranium to stainless steel. MARK-1A subassemblies were used during most of the period in which the samples resided in core; therefore the depleted uranium in the blanket zone of the simplified model was replaced by stainless steel (identical with the reflector). In order to check the adequacy of both configurations, two models have been calculated, one with depleted uranium in the core outer region and the other with stainless steel.

The calculations were performed with the modular AMPX9 code system, from which the modules AJAX, BONAMI, XSDRN, and MALOCS were utilized. Cross sections were taken from the 174-group VITAMIN-E library which is based on the recently released ENDF/B-V. One of the goals of this 
study is to check this library against the former EMUF/B-IV library sinre at the time there was little experience with fast reactor calculations utilizing ENDF/B-Y.

The calculational procedure starts with a resonance self-shielding calculation for the mixtures in the core and the blanket. Assuming the materials in each region were homogenously mixed, the resonance self-shielding calculation was performed by the Bondarenko method with the BONAMI module. As might have been expected, the self-shielding effect was negligibly small because the EBR-II has such a hard spectrum that most of the neutrons never reach the resonance region. To demonstrate this fact, shielded and unshielded on -group cross sections collapsed from the ViTAMIN-E library are given in Table 4.2. The collapse was performed in two steps: first using a typical ZPR spectrum, the 174-group cross sections were collapsed into a 15-group data set; then using the EBR-II spectrum obtained from ANL, the 15group cross sections were further collapsed into a one-group data set.

Table 4.2 A Comparison Between Resonance Self-shielded and Unshielded One-Group Cross Sections (in barns)

\begin{tabular}{|c|c|c|c|c|}
\hline \multirow{2}{*}{ Nuclide } & \multicolumn{2}{|c|}{$\sigma_{c}$} & \multicolumn{2}{|c|}{$\sigma_{f}$} \\
\hline & Unshielded & Shielded & Unshielded & Shielded \\
\hline $235 U$ & .2444 & .2443 & 1.3407 & 1.3403 \\
\hline $238 \mathrm{U}$ & .1397 & .1383 & .0799 & .0799 \\
\hline $239 \mathrm{Pu}$ & .1535 & .1533 & 1.6559 & 1.6557 \\
\hline
\end{tabular}


following the self-shielding calculations, the flux distributions for the two previously mentioned EBR-II models were computed with the onodimensional discrete ordinate transport code XSDRN-PM. In the present calculations an $S_{8}-P_{3}$ approximation was used and as many as 49 intervals were used for the spatial distribution. The same code was also utilized to collapse the cross sections from 174 groups to one group in each of the subregions of the two driver cores. It should be noted that on!y the cross sections for the most important isotopes have heen selected for collapsing in this way. For all ther isotopes a generic one-group library for LMFBR calculations was used in subsequent depletion calculations.

In Table 4.3, one-group cross sections for capture, fission, and $(n, 2 n)$ reactions are given for the 3 subregions of the uranium blanketed driver core. Similar cross sections for the stainless steel blanketed core are given in Table 4.4, however, with a slight difference. Since cross sections in zones 1 and 2 are almost the same, Table 4.4 does not contain cross sections for zone 1 . On the other hand, since the spectrum in the stainless steel is much softer than in the core, it is expected that the spectrum at the core outer Doundary will also be softer. For tinis reason an additional subzone, which is the last interval of the driver core, was added to Table 4.4 designated as zone $3^{-}$. Note that this zone is a part of zone 3 and that the cross sections for zone 3 are averaged over a core section also containing zone $3^{\circ}$. 


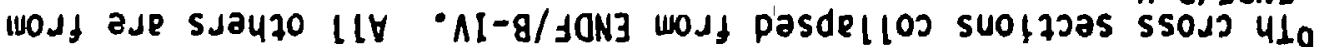

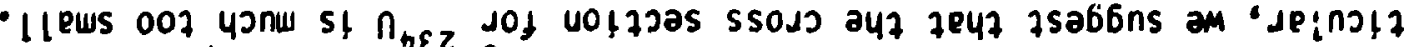

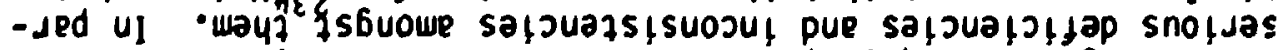

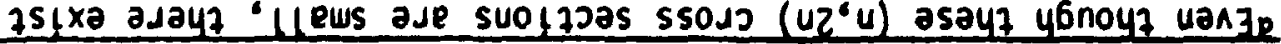

\begin{tabular}{|c|c|c|c|c|}
\hline$\varepsilon-982^{\circ} \downarrow$ & $080^{\circ} z$ & $1622^{\circ}$ & $\varepsilon$ & \\
\hline$\varepsilon-2 \varepsilon O^{\circ} s$ & $\$ 9 L^{\circ} I$ & $896 I^{\circ}$ & 2 & \\
\hline$\varepsilon-88 I^{\circ} \mathcal{G}$ & $90 L^{*} I$ & $\varepsilon 681^{\circ}$ & $\mathfrak{l}$ & ndinz \\
\hline $0-688^{\circ} Z$ & $I-\log ^{\bullet} 9$ & SOS2 & $\varepsilon$ & \\
\hline$\nabla-26 \varepsilon^{\circ} \varepsilon$ & $I-6 t I \cdot l$ & SEOZ` & 2 & \\
\hline$D-\angle 6 b^{\circ} \varepsilon$ & $I-\varepsilon \varepsilon \varepsilon^{\bullet} \iota$ & $\angle \varepsilon 6 I^{\circ}$ & $\mathfrak{l}$ & ndonz \\
\hline$b-859 \cdot 9$ & $089^{\circ} 1$ & S68 ${ }^{\circ}$ & $\varepsilon$ & \\
\hline$t-00 L^{\circ} L$ & $699^{\circ} 1$ & $09 b I^{\circ}$ & 2 & \\
\hline$t-6 \varepsilon 6^{\circ} L$ & $699^{\circ} I$ & $69 \varepsilon I^{\circ}$ & I & $n_{d} \in \varepsilon$ \\
\hline$\varepsilon-090^{\circ} \varepsilon$ & $z-0 L L^{\bullet} 8$ & $\operatorname{LOSI} I^{\bullet}$ & $\varepsilon$ & \\
\hline$\varepsilon-\varepsilon 6 \varsigma^{\circ} \varepsilon$ & $I-010^{\bullet} I$ & 9ZEI॰ & 2 & \\
\hline$\varepsilon-\mathcal{S O} L^{\circ} \varepsilon$ & $I-2 b 0^{\circ} I$ & $E \angle Z I^{\circ}$ & I & $n_{8 \varepsilon z}$ \\
\hline$\varepsilon-\angle 99^{\circ} I$ & $\varepsilon 6 \varepsilon^{\bullet} I$ & $9\left[\angle Z^{\circ}\right.$ & $\varepsilon$ & \\
\hline$\varepsilon-0 t 8^{\circ} \mathrm{I}$ & $D E E \cdot I$ & ५ZદZ• & 2 & \\
\hline$\varepsilon-\angle 68^{\circ} I$ & $0 Z E^{\bullet} I$ & $9222^{\circ}$ & I & nsEz \\
\hline$b-26 \varepsilon^{\circ} \varepsilon$ & $I-\$ 60 \cdot 9$ & $98 z \varepsilon^{\circ}$ & $\varepsilon$ & \\
\hline$t-i 6 t^{\circ} \varepsilon$ & $I-b I L^{\bullet} g$ & I682 & 2 & \\
\hline$\downarrow-โ \varsigma 0^{\circ} \varepsilon$ & $I-068^{\circ} 9$ & $6182^{\circ}$ & I & PHEZ \\
\hline$\varepsilon-660^{\circ} \tau$ & $29 I^{\circ} 2$ & $0 \angle S I^{\circ}$ & $\varepsilon$ & \\
\hline$\varepsilon-\tau 62 \cdot \tau$ & $\angle 80^{\circ} \mathrm{Z}$ & S6EI• & 2 & \\
\hline $\mathcal{E}-\left\lfloor\varepsilon \varepsilon^{*} โ\right.$ & $1 \angle 0^{\circ} 2$ & ESEI• & I & $n_{\varepsilon \varepsilon 乙}$ \\
\hline$\varepsilon-866^{\circ} z$ & $026^{\circ} \mathrm{I}$ & $\angle Z 12^{\circ}$ & $\boldsymbol{\varepsilon}$ & \\
\hline pazelnoles q0u & $z-02 z^{\circ} z$ & $8981^{\circ}$ & 2 & \\
\hline$\varepsilon-\varepsilon \angle 9^{\circ} \varepsilon$ & $062^{\circ} 2$ & I08I• & I & 94J.2EZ \\
\hline 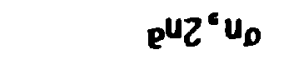 & to & $J_{0}$ & auoz & adozosI \\
\hline
\end{tabular}

zayue lg wn!uedn pazalda e yz!m lapow

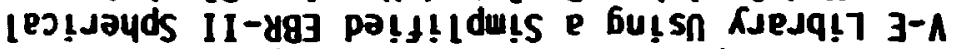

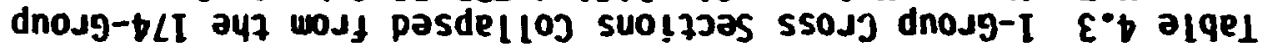


Table 4.4 1-Group Cros: Sections Collapsed from the 174-Group I-E Library Using a Simplified EBR-II Spherical

Model with a Stainless Steel Blanket

\begin{tabular}{|c|c|c|c|c|}
\hline Isotope & Zone & $\sigma_{C}$ & $\sigma f$ & $\sigma_{n, 2 n^{a}}$ \\
\hline \multirow[t]{3}{*}{${ }^{233} U$} & 2 & .1409 & 2.093 & $1.280-3$ \\
\hline & 3 & .1763 & 2.271 & $1.098-3$ \\
\hline & $3^{\prime}$ & .2264 & 2.539 & $8.932-4$ \\
\hline \multirow[t]{3}{*}{$234 \mathrm{U}$} & 2 & .2930 & $6.694-1$ & $3.552-4$ \\
\hline & 3 & .4366 & $6.089-1$ & $3.046-4$ \\
\hline & $3^{\prime}$ & .6887 & $5.553-1$ & $2.479-4$ \\
\hline \multirow[t]{3}{*}{$235 U$} & 2 & .2344 & 1.3368 & $1.8 \hat{c} 5-3$ \\
\hline & 3 & .2938 & 1.436 & i.564-3 \\
\hline & $3^{\prime}$ & .3676 & 1.569 & $1.273-3$ \\
\hline \multirow[t]{3}{*}{$238 \mathrm{U}$} & 2 & .1333 & $1.003-1$ & $3.564-3$ \\
\hline & 3 & .1569 & $8.758-2$ & $3.055-3$ \\
\hline & $3^{\prime}$ & .1843 & $7.480-2$ & $2.486-3$ \\
\hline \multirow[t]{3}{*}{$239 \mathrm{Pu}$} & 2 & .1490 & 1.671 & $7.636-4$ \\
\hline & 3 & .2426 & 1.742 & $6.548-4$ \\
\hline & $3^{\prime}$ & .3831 & 1.876 & $5.238-4$ \\
\hline \multirow[t]{3}{*}{$240 \mathrm{Pu}$} & 2 & .2077 & $7.127-1$ & $3.364-4$ \\
\hline & 3 & .4453 & $6.498-1$ & $2.885-4$ \\
\hline & $3^{\prime}$ & 1.0107 & $5.934-1$ & $2.347-4$ \\
\hline \multirow[t]{3}{*}{$241 \mathrm{Pu}$} & 2 & .1994 & 1.771 & $4.991-3$ \\
\hline & 3 & .2718 & 1.970 & $4.279-3$ \\
\hline & $3^{\prime}$ & .3791 & 2.277 & $3.482-3$ \\
\hline
\end{tabular}

Even though these $(n, 2 n)$ cross sections are small, there exist serious defictenctes and inconsistencies amongst them. In particular, we suggest that the cross section for ${ }^{234} \mathrm{U}$ is much too small. 
Upon examining the data in Tables 4.3 and 4.4 , one notices first that there are only slight differences between cros- sections in zones 1 and 2 of both models (this is true also for the cross sections of zone 1 in the stainless steel blanketed reactor not given in Table 4.4). Consequently it was concluded that one-group cross sections are insensitive to the composition of the blanket and to the location in the core over a region which spieads far away from the core center. Since many of the samples were located in this region, we may be fairly confident about the spectrum-averaged cross sections used in the depletion calculations for these samples. However, the rapid increase in the capture cross sections of zone 3 in both models and the high cross sections obtained at the core-reflector interface of the core with the stainless steel blanket indicate sensitivity to modeling approximations in cross sections for samples at these locations. The effect of the reactor location on the flux spectrum is shown in Fig. 3, where flux spectra at the core center and at the core outer boundary are plotted. From this figure it can be seen that the spectrum at the core center is harder than the spectrum at the outer boundary. This is the reason for the capture cross section increase at off-center areas as shown in Tables 4.3 and 4.4 . 
Figure 3. Calculated flux Spectra at the Core Center and the Core Outer Boundary of EBR-II

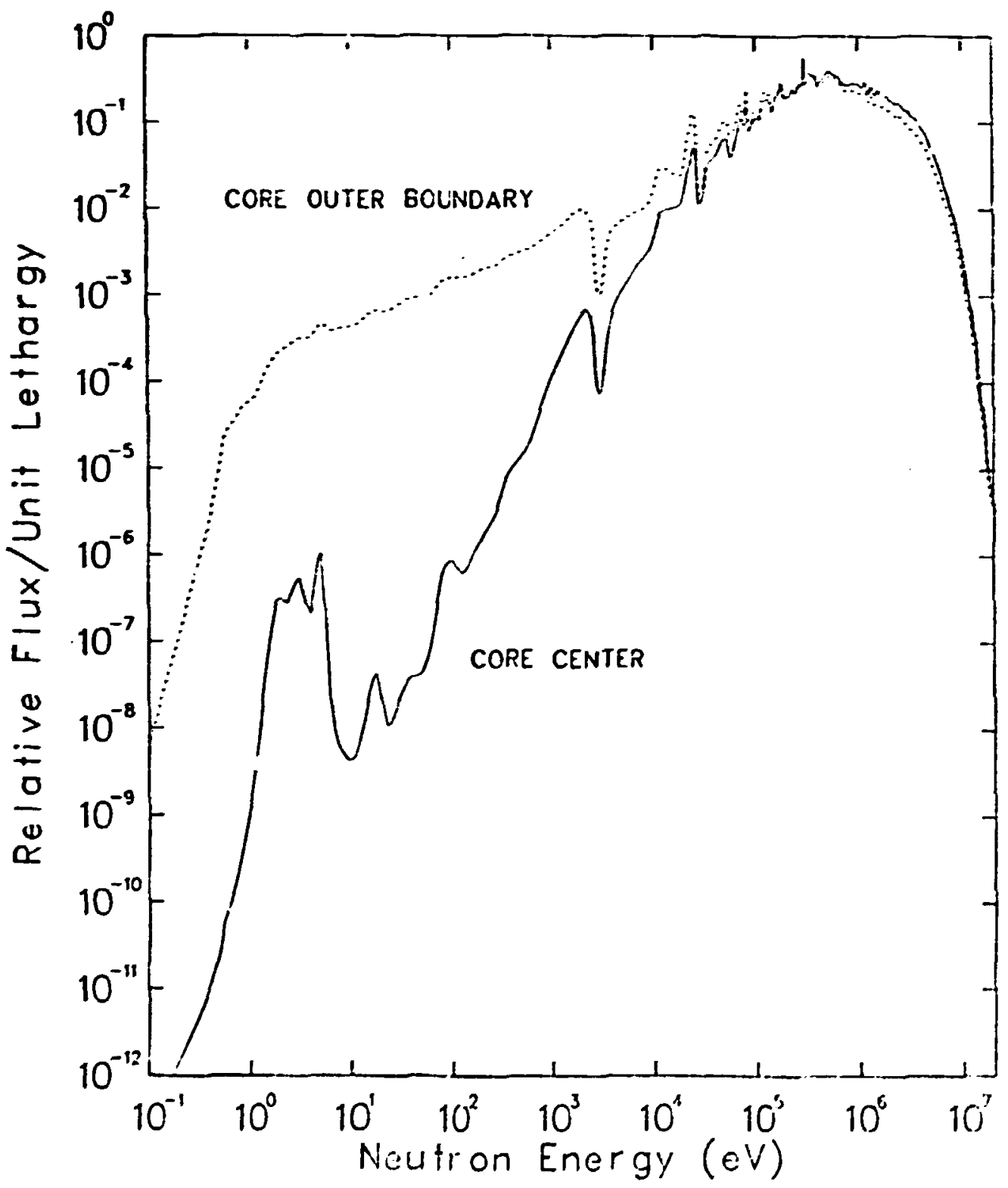


As already mentioned, one goal of this study was to test results based on EMDF/B-V cross sections against results wich mere derived using ENDF/B-IV. To that end the EBR-II model with the depleted uranium blanket was calculated with an older 174-group library based on ENDF/B-IV and one-group cross sections were processed the same way as previously. In the next two tables, capture and absorption cross sections obtained with both libraries are compared. As may be seen, ENDF/B-V cross sections usually differ by less than $2.5 \%$ from the ENDF/B-IV values with an exception for $232 \mathrm{Th}$ and $240 \mathrm{Pu}$ (capture and absorption) and $234 \mathrm{U}$ (absorption only). The differences in the thorium cross sections are thought to result from a known error in the ENDF/B-V file, so that for this nuclide, ENDF/B-IV cross sections are preferred. Differences in the other cross sections presumably reflect improvements arising from new measurements and/or evaluations. 
Table 4.5. Comparison Between ERDF/B-IV and V Capture Cross Sections

\begin{tabular}{|c|c|c|c|c|}
\hline Isotope & Zone & $\begin{array}{c}\text { ENDF/B-IV } \\
\sigma_{C}\end{array}$ & $\begin{array}{c}\text { ENDF } / B-Y \\
\sigma_{C}\end{array}$ & $\begin{array}{l}\text { Relative } \\
\text { Change (x) }\end{array}$ \\
\hline \multirow[t]{3}{*}{$232 \mathrm{Th}$} & 1 & .1801 & . 1599 & -11.2 \\
\hline & 2 & .1858 & \multicolumn{2}{|c|}{ not calculated } \\
\hline & 3 & .2127 & .1779 & -16.4 \\
\hline \multirow[t]{3}{*}{$233 \mathrm{U}$} & 1 & .1374 & .1353 & -1.5 \\
\hline & 2 & .1415 & .1395 & -1.4 \\
\hline & 3 & .1581 & .1570 & -0.7 \\
\hline \multirow[t]{3}{*}{$234 U$} & 1 & .2821 & .2813 & -0.2 \\
\hline & 2 & .2893 & .2891 & -0.1 \\
\hline & 3 & .3253 & .3286 & 1.0 \\
\hline \multirow[t]{3}{*}{$235 U$} & 1 & .2283 & .2226 & -2.5 \\
\hline & 2 & .2383 & .2325 & -2.4 \\
\hline & 3 & .2784 & .2716 & -2.4 \\
\hline \multirow[t]{3}{*}{$238 \mathrm{U}$} & 1 & .1310 & .1279 & -2.4 \\
\hline & 2 & .1357 & .1326 & -2.3 \\
\hline & 3 & .1537 & .1501 & -2.3 \\
\hline \multirow[t]{3}{*}{$239 \mathrm{Pu}$} & 1 & .1403 & .1369 & -2.3 \\
\hline & 2 & .1494 & .1460 & -2.3 \\
\hline & 3 & .1927 & .1895 & -1.7 \\
\hline \multirow[t]{3}{*}{$240 \mathrm{Pu}$} & 1 & .1757 & .1937 & 10.2 \\
\hline & 2 & .1846 & .2035 & 10.2 \\
\hline & 3 & .2278 & .2505 & 10.0 \\
\hline \multirow[t]{3}{*}{$241 \mathrm{Pu}$} & 1 & .1918 & .1893 & -1.3 \\
\hline & 2 & . 1993 & .1968 & -1.3 \\
\hline & 3 & .2318 & .2291 & -1.2 \\
\hline
\end{tabular}


Table 4.6. Comparison Between ErDF/B-IV and V Abserption Cross Sections

\begin{tabular}{|c|c|c|c|c|}
\hline Isotope & Zone & $\begin{array}{c}\text { ENDF/B-IV } \\
\sigma_{a}\end{array}$ & $\begin{array}{c}\text { ERDF } / B-V \\
\sigma_{\mathbf{a}}\end{array}$ & $\begin{array}{l}\text { Relative } \\
\text { Change }(\$)\end{array}$ \\
\hline \multirow[t]{3}{*}{$232 \mathrm{Th}$} & i & 0.2030 & 0.1839 & -9.4 \\
\hline & 2 & 0.2080 & \multicolumn{2}{|c|}{ not calculated } \\
\hline & 3 & 0.2319 & 0.1983 & -14.5 \\
\hline \multirow[t]{3}{*}{$233 v$} & 1 & 2.1639 & 2.2062 & 2.0 \\
\hline & 2 & 2.1872 & 2.2266 & 1.8 \\
\hline & 3 & 2.2882 & 2.3191 & 1.4 \\
\hline \multirow[t]{3}{*}{$234 \mathrm{U}$} & 1 & 0.9037 & 0.9707 & 7.4 \\
\hline & 2 & 0.8946 & 0.9604 & 7.4 \\
\hline & 3 & 0.8731 & 0.9381 & 7.4 \\
\hline \multirow[t]{3}{*}{$235 U$} & 1 & 1.5597 & 1.5424 & -1.1 \\
\hline & 2 & 1.5843 & 1.5662 & -1.1 \\
\hline & 3 & 1.6878 & 1.6642 & -1.4 \\
\hline \multirow[t]{3}{*}{$238 U$} & 1 & 0.2314 & 0.2321 & 0.2 \\
\hline & 2 & 0.2331 & 0.2336 & 0.2 \\
\hline & 3 & 0.2383 & 0.2378 & -0.2 \\
\hline \multirow[t]{3}{*}{$239 \mathrm{Pu}$} & 1 & 1.7961 & 1.8055 & 0.5 \\
\hline & 2 & 1.8052 & 1.8147 & 0.5 \\
\hline & 3 & 1.8601 & 1.8949 & 1.9 \\
\hline \multirow[t]{3}{*}{$240 \mathrm{Pu}$} & 1 & 0.8767 & 0.9270 & 5.7 \\
\hline & 2 & 0.8681 & 0.9184 & 5.8 \\
\hline & 3 & 0.8489 & 0.9006 & 6.1 \\
\hline \multirow[t]{3}{*}{$241 \mathrm{Pu}$} & 1 & 1.9451 & 1.9352 & -0.5 \\
\hline & 2 & 1.9705 & 1.9621 & -0.4 \\
\hline & 3 & 2.0858 & 2.0801 & -0.3 \\
\hline
\end{tabular}




\section{THE TIME-DEFENDENT PROBLEM: BUILDUP AND DECAY OF TRANSHTATION PRODUCTS}

Having obtained appropriate space-dependent cross sections for each sample location, the time-dependent transmutation process by which nuclides are generated and destroyed may be described by the following matrix equation: 10

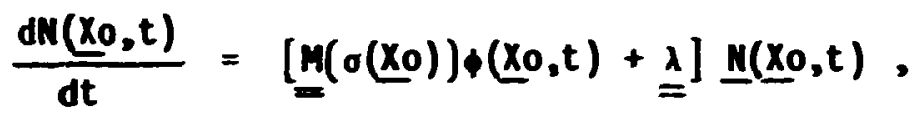

where $M\left(\sigma\left(x_{0}\right)\right)$ is a time-independent matrix which contains all (one-group) cross sections that contribute to the transmutation of nuclides at position $\underline{x}_{0}, \underline{\lambda}$ is a matrix of radioactive decay constants, including $a, B^{-}$, and $B^{+}$decays as well as appropriate branching ratios, and $\left(x_{0}, t\right)$ is the total energy-integrated flux at the reactor location $\underline{x}_{0}$. He have chosen to write Eq. (1) in a somewhat unconventional way in order to emphasize some aspects of the approach used in the present study. First, the space-time separation becomes apparent in view of the fact that the cross section matrix $M\left(\sigma\left(X_{0}\right)\right)$ is considered to be time-independent. Further the flux at point $\underline{x}_{0}$ is usually a complex function which depends on the core composition, burnup level, control rod position and reactor power. However, as was already stated, we assume that the flux may be separated in space and time so that we may write:

$$
\phi(x, t)=\phi_{0}(x) \cdot x(t)
$$

We may further express the spatial term $\phi_{0}(x)$ as a fraction or the maximum flux: 


$$
\varphi_{0}(x)=\varphi_{0}(\max ) \cdot \phi(x),
$$

where $\psi(x)$ may be regarded as a shape function of the flux, independent of time and flux level.

Inserting Eqs. (2) and (3) into (1), we obtain for the nuclides at position $x_{0}$ :

$$
\frac{d \underline{M}\left(\underline{x}_{0}, t\right)}{d t}=\left[\phi_{0}(\max ) \underline{M} \sigma\left(\underline{x}_{0}\right) \forall\left(\underline{x}_{0}\right) x(t)+\underline{\underline{\lambda}}\right] \underline{M}\left(\underline{x}_{0}, t\right) .
$$

Under the assumption that the flux is separable in tire and space, Eqs. (1) and (4) are equivalent. The advantage of Eq. (4) is that it expresses the space and time dependence of the flux in easily measurable quantities. The value for $\phi_{0}(\max )$ was measured in EBR-II and was found to be $2.5 \cdot 10^{15} / \mathrm{cm}^{2} \mathrm{~s}$ at core center when the reactor was operating at 50rw. The shape function may be either measured or calculated, and the time function $x(t)$ may be obtained from the recorded power history of the reactor, since by constant core characteristics, the time dependence of the flux at any location is proportional to the power output of the reactor. It should be noted however, that when the core suffers substantial burnup, this statement is no longer valid because in that case higher fluxes are required in order to maintain the reactor power at a constant level. However, since burnups of only 1-2\% were recorded 11 for representative subassemblies, power and flux may be assumed proportional over the entire considered irradiation period.

The irradiation of the samples began on vecenber 6,1965 when run number 24 was started, and ended on September 10, 1970 with the shutdown of run 458. During this time the reactor was shut down many 
tines. However, although a full record of the power schedule was provided by ANL, the history of the samples during this period was not well documented, a fact wich caused some confusion in preliminary calculation. For that reason a full description of the irradiation history of the samples is given in Appendix A, indicating primarily two major events: first, the subassembly wich contained the samples was removed from its original location at row 2 (one lattice position off center) to row 4 before starting run $34 A$; anc secondly, there were several runs when the sampies were entirely withdrawn from the reactor. These two events significantly affected the total fluence to which the samples were exposed and vere taken into account in the subsequent depletion calculations.

To complete the description of the irradiation history, the flux level at each sample's location must be given. Once a flux distribution for any power level is given, it may be used to obtain a shape function from which fluxes at any location may be calculated. As already stated, such a distribution, factorized in axial and radial functions, was provided by ANL. Thus an overall shape function could be determined as a product of the axial and radial shape functions, each normalized to 1.0 at the reactor center. The radial distribution showed that at row 2 the total flux was 0.99 of the flux at core center, whereas at row 4 it dropped to 0.85 . The axial shape factor for the different samples is given in Table 4.7. However, since the flux distributions provided by ANL exterid over the driver core only, shape factors for the samples in the lower axial blanket may not be given, and therefore calculations were not performed for thcse samples. In Table 4.7 the slight asymmetry between top and bottom should be noted. 
Table 4.7. Axial Shape Factors for Samples in Driver Core

\begin{tabular}{cccc}
\hline $\begin{array}{c}\text { Sample } \\
\text { Position }\end{array}$ & $\begin{array}{c}\text { Sample } \\
\text { ID }\end{array}$ & $\begin{array}{c}\text { Major } \\
\text { Isotope }\end{array}$ & $\begin{array}{c}\text { Axial } \\
\text { Shape Factor }\end{array}$ \\
\hline 1 & 21 & $232 \mathrm{Th}$ & .73 \\
2 & 81 & $238 \mathrm{U}$ & .81 \\
3 & 92 & $239 \mathrm{U}$ & .89 \\
4 & 31 & $233 \mathrm{~J}$ & .94 \\
5 & 53 & $235 \mathrm{U}$ & .97 \\
6 & 93 & $239 \mathrm{U}$ & 1.00 \\
7 & 51 & $235 \mathrm{U}$ & 1.00 \\
8 & 94 & $239 \mathrm{Pu}$ & .97 \\
9 & 32 & $233 \mathrm{U}$ & .95 \\
10 & 02 & $240 \mathrm{Pu}$ & .89 \\
11 & 14 & $241 \mathrm{Pu}$ & .82 \\
12 & 72 & $237 \mathrm{~Np}$ & .74 \\
\hline
\end{tabular}

To perform the time-dependent depletion calculations, the code ORIGEN ${ }^{10}$ was utilized. Given a set of initiai values for the nuclides of interest and a set of one-group cross sections, this code traces the process of formation and destruction of these nuclides for any giver flux history. Initial values and flux history are provided as input to the code whereas the cross-section and decay constant matrices are usuaily taken from standard libraries. An option provided in ORIGEN, however, allows cross sections to be input and therefore those generated by the simplified EBR-II model calculations were used to replace tine standard ORIGEN library. The library was altered from sample to sample according to their position in the reactor. 
A special option in ORIGEN enables the calculation of transmutation processes after shuzdown. This option was used to extend the calculations over the postirradiation period till the time the samples were finally analyzed, a period of 8-10 years in most cases. The addition of this period to the calculations is important not only for the unstable nuclides, but also for stable ones wich mav gain appreciable amounts during this period from the decay of other nuclides. As an example, 10 years after withdrawal from the reactor the $238 \mathrm{Pu}$ content in a $240 \mathrm{pu}$ sample has increased by about $25 \%$ because of the a decay of $242 \mathrm{Am}$.

Calculational results will be given in the next section and compared to experimentally determined ralues. 
36 


\section{COMPARISON OF CALCULATIONAL AND EXPERIMENTAL RESULTS}

In this chapter we will finally turn to the actual calculational and experimental results. Out of the 12 samples which have been irradiated in the driver core, 10 were "completely analyzed," by wich we mean that compositions of both the irradiated and unirradiated samples were measured. The two remaining samples were not measured because duplicate samples were available.

The calculations were performed using cross sections and fluxes as described above. However, in order to check the a priori validity of our EBR-II simplified model, some basic parameters related to this model were compared with measured ones. Agreement between these parameters will help to el iminate sources of uncertainties in case discrepancies between measured and calculated exposure products occur. He will therefore concentrate first in a comparison between measured and calculated [BR-II parameters; then we will discuss the results obtained for the irradiated samples.

\section{A. COMPARISON BETWEEN CALCULATED AND MEASURED PARAMETERS OF EBR-II}

We have shown above that the flux in the central part of the EBR-II model is essentially asymptotic and is insensitive to changes to the outer core environment. For this reason, calculated and measured data are expected to agree best for the innermost subzone of the driver core. The most obvious way to check this agreement is to compare calculated and experimsntal flux spectra; yet a measured flux spectrum unfortunately was not avallable. Instead we compared the calculated 
174-group flux spectrun with a spectrum computed by ANL from a twodimensional model which is believed to represent the reactor configuration more accurately than our 1-dimensional spherical model. The AIL calculations were carried out with the 2-D transport code DOT, utilizing a 22 group cross-section library based on an early ENDF/B file. In order to compare flux spectra, the 174-group spectrum was condensed into a 15-group spectrum with a group structure corresponding to that of the ANL spectrum. It was found, however, that 11 groups might be sufficient for the comparison of both spectra because for the low energy groups (E $<0.68 \mathrm{eV}$ ) the flux is negligible. Both spectra are given in Table 5.1 from which it can be seen that for most important groups the values agree to about $10 \%$. Differences of this magnitude can be caused by the difference in cross sections used by ORNL and ANL. It is especially significant that the ENDF/B-V fission spectrum used by ORNL is considerably harder than the older spectrum used by ANL.

Table 5.1. ANL and ORNL 14-Group Flux Spectra for EBR-II

\begin{tabular}{ccccc}
\hline Group & $\begin{array}{c}\text { Upper } \\
\text { Energy(MeV) }\end{array}$ & $\begin{array}{c}\text { ANL Flux } \\
\text { Spectrum }\end{array}$ & $\begin{array}{c}\text { ORNL Flux } \\
\text { Spectrum }\end{array}$ & $\begin{array}{c}\text { Relative } \\
\text { Change( })\end{array}$ \\
\hline 1 & 10.000 & 0.074 & 0.111 & 50.0 \\
2 & 2.231 & 0.087 & 0.095 & 9.2 \\
3 & 1.353 & 0.120 & 0.132 & 10.0 \\
4 & $8.209-1$ & 0.341 & 0.328 & -3.8 \\
5 & $3.020-1$ & 0.245 & 0.214 & -12.7 \\
6 & $1.111-1$ & 0.098 & 0.086 & -12.2 \\
7 & $4.087-2$ & 0.027 & 0.027 & 0.0 \\
8 & $1.503-2$ & $6.0-3$ & $5.768-3$ & -3.9 \\
9 & $5.531-3$ & $7.0-4$ & $5.257-4$ & -24.9 \\
10 & $3.355-3$ & $2.0-4$ & $1.526-4$ & -23.7 \\
11 & $2.035-3$ & $4.0-4$ & $3.104-4$ & -22.7 \\
\hline
\end{tabular}


A further parameter wich might be considered is the central capture-to-fission ratio, which is the ratio of the spectrum-averaged one-group capture cross section to the average fission cross section at the core center. Experimental as well as calculational values for various isotopes were reported in Ref. 4 and are compared with data obtained from Table 4.3. This is shown in Table 5.2, which shows fairly good agreement between ratios calculated with the EBR model and those determined experimentally. Deviations between ORNL and ANL values are of the order of a few percent only, except for the ANL calculated value for $242 \mathrm{Pu}$, which seems to be unreasonable.

Table 5.2. Experimental vs. Calculated Capture-to-Fission Ratios for Various Isotopes

\begin{tabular}{ccccccc}
\hline & \multicolumn{2}{c}{ ANL } & & \multicolumn{2}{c}{ ORNL } \\
\cline { 2 - 5 } \cline { 5 - 7 } Nuclide & Measured & Calculated & C/E & Calculated & /E \\
\hline $233 \mathrm{U}$ & .073 & .069 & .945 & .065 & .890 \\
$235 \mathrm{U}$ & .180 & .161 & .894 & .169 & .939 \\
$239 \mathrm{Pu}$ & .085 & .080 & .941 & .082 & .965 \\
$240 \mathrm{Pu}$ & .305 & .277 & .908 & .264 & .866 \\
$242 \mathrm{Pu}$ & .469 & .208 & .443 & $.419^{\star}$ & .893 \\
\hline
\end{tabular}

* From standard ORIGEN Library.

The results in Table 5.2 establish the expected agreement between experimental and calculated parameters of EBR-II at the core center and verify the adequacy of the spherical model. It might, 
however, be also interesting to examine some space-dependent characteristic of the reactor in order to assess the validity of the coaputed space-dependent spectra. To that end we examined the spatial distributions of capture-to-fission ratios of various isotopes wich have been irradiated in EBR-II.12 This experiment took place in the early days of EBR-II (prior to the actinide samples experiment) when the core was fueled with MARK-I type subassemblies in seven rows only and had no reflector. Such a reactor setup may be represented by a spherical model better than any other configuration since there are only two compositions to be considered: the driver core and the depleted uranium blanket. In the experiments reported, many samples were placed at various distances from the vertical axis, in or next to the horizontal midplane of the core. Assuming azimuthal symmetry of the reactor, the radial direction in the horizontal midplane may be adequately represented by the only coordinate of the spherical model, so that a good agreement between calculation and model might be expected in spite of all limitations of the calculational model. In Figure 4, calculational and experimental results for four isotopes irradiated at various locations are shown. The experimental data were taken from Ref. 12, whereas our calculational results are from a two-region spherical model of EBR-II with an inner core divided in 5 subzones. The calculated capture-to-fission ratios of each subzone shown in Figure 4 are volume averaged, but the overall agreement between experimental and calculational results is still 


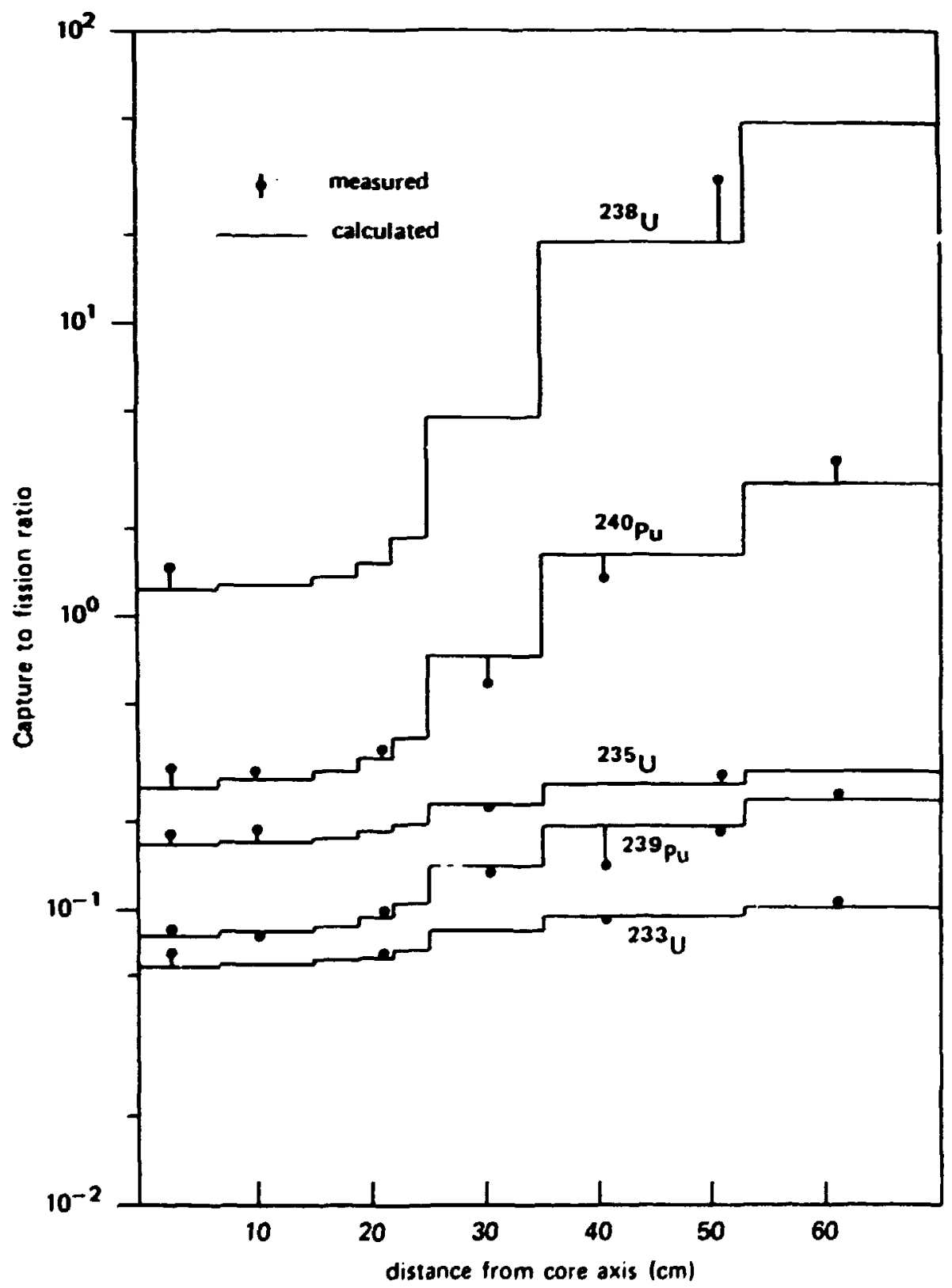

Fig. 4. Capture-to-fission Ratio Distrihution in Horizontal Midplane of EBR-II. 
surprisingly good, even at locations deep in the blanket. We may therefore conclude that when correctly interpreted, simple models may give very acceptable results. However, since the actinide :amples were arranged in a vertical direction, successful application sf a simple spherical model for their analysis is not assured.

The overall conclusion of this section is that the simplified spherical model will produce acceptasle results for samples irradiated near the horizontal midplane of the reactor, but for samples located near the axial blanket, calculational results are expected to be less accurate, due both to inaccuracies in the spectrum calculation and to uncertainties in determining an "equivalent" location for the shape factor.

B. CALCULATED AND mEASUREd EXPOSURE PRODUCTS IN THE ACTINIDE SAMPLES In this section, calculated and measured values for the exposure products in the actinide samples will be given. The calculations are based on the spherinal three-zone model composed of a driver core surrounded by a reflector and a "blanket," both made of stainless steel. This model was chosen because it is believed to correspond best to the reactor composition in the vertical direction during the major part of the irradiation period. When the actinide samples were introduced into the reactor, MARK-IA type fuel subassemblies having stainless steel axial blankets were being used.

To start the calculation, the initial composition of the samples must be known. These were determines experinentally using control samples with compositions identical to those of the irradiated samples. 
Most of these samples, however, have been analyzed at about the same time as the irradiated ones; therefore the actual composition at the initial time of irradiation must be calculated backwards for isotopes with relatively short half lives. Such a calculation was especially necessary for $241 \mathrm{Pu}$, wich has a half life of 14.3 years.

The initial composition for the various samples, corrected to the initial time of irradiation, are given in Table 5.3. With the exception of the ${ }^{239} \mathrm{Pu}$ samples, the data in Tahle 5.3 results from measurements made at ORML at about mid-1980. The relative abundance of the isotopes in the $239 \mathrm{Pu}$ sample (\$93) was measured by ANL in 1974 and is valid also for the two other ${ }^{239} \mathrm{Pu}$ samples (\$92 and 94). Two samples are not indicated: the $232 \mathrm{Th}$ sample of $32.81 \mathrm{mg}$ and the ${ }^{237} \mathrm{~Np}$ sample of $1.0 \mathrm{mg}$. These two samples are assumed to be nearly $100 \%$ pure.

Table 5.3. Initial Composition of Actinide Samples Irradiated in EBR-II (in $\mathrm{mg}$ )

\begin{tabular}{l|l|l|l|l|l|l}
\hline \multirow{2}{*}{ SAMPLE } & $\# 81$ & $\# 31^{\mathrm{a}}$ & $\# 53$ & $\# 94 \mathrm{~b}$ & $\# 02$ & $\# 14$ \\
\cline { 2 - 7 } & $238 \mathrm{U}$ & $233 \mathrm{U}$ & $235 \mathrm{U}$ & $239 \mathrm{Pu}$ & $240 \mathrm{Pu}$ & $241 \mathrm{Pu}$ \\
\hline $\begin{array}{l}233 \mathrm{U} \\
234 \mathrm{U} \\
235 \mathrm{U}\end{array}$ & & $9.859+1$ & & & & \\
$236 \mathrm{U}$ & $9.160-4$ & 1.052 & $7.968-1$ & & & \\
$238 \mathrm{U}$ & $4.120-2$ & $1.929-2$ & $8.218+1$ & & & \\
$238 \mathrm{Pu}$ & $1.650-3$ & $9.639-4$ & $3.858-1$ & & & \\
$239 \mathrm{Pu}$ & $9.154+1$ & $4.125-1$ & 4.825 & & & \\
$240 \mathrm{Pu}$ & & & & & & \\
$241 \mathrm{Pu}$ & & & & $1.006+2$ & $4.240-1$ & $3.727-1$ \\
$242 \mathrm{Pu}$ & & & & 4.857 & $5.083+1$ & 1.335 \\
& & & & $3.460-1$ & $2.504-1$ & $3.069+1$ \\
\hline
\end{tabular}

afor $23.3 \mathrm{U}$ sample 32 multiply all values by 1.344 .

bFor $239 \mathrm{Pu}$ sample $" 93$ multiply all values by 1.295 . 
In Table 5.4 measured quant: ties of the exposure products in 10 samples are given. Four of these samples, namely 233U(\$32), 235U(\$53), ${ }^{239} \mathrm{Pu}(\$ 93)$ and $24 \mathrm{LPu}(\$ 14)$, were measured by $\mathrm{NL}$; the other ones were measured at ORNL.

Now we turn to the calculated values. Using the initial compositions from Table 5.3 and appropriate cross sections and flux levels, the exposure products in each sample were calculated with the ORIGEN code. These calculations span a period of nearly 15 years, including the irradiation period as well as the out-of-core decay period, Caiculations were performed for 7 samples only: each pair of the ${ }^{233} \mathrm{U}$ and $239 \mathrm{Pu}$ samples required one calculation only since the samples of the same type had, up to a constant factor, the same initial composition. The slight differences in the flux factor may be accounted for accurately by a linear adjustment, although the burnup process is a strictly nonlinear function of the flux level. For the ${ }^{237} \mathrm{~Np}$ sample, a hand calculation was sufficient to obtain values for the tho measured nuclides of this sample. Calculated results for all samples a.e given in Table 5.5 .

With the results of Tables 5.4 and 5.5 one may compare the calculation against the experiment. One way to carry out such a comparison is simply to calculate the ratios of the calculated to the experimentally obtained quantities $(C / E)$ of the end products. One, of course, desires these ratios to be as close as possible to $1 . \hat{v}$, but even so, this might not necessarily indicate a good correlation between calculation and experiment. The reason for that lies in the fact that the 


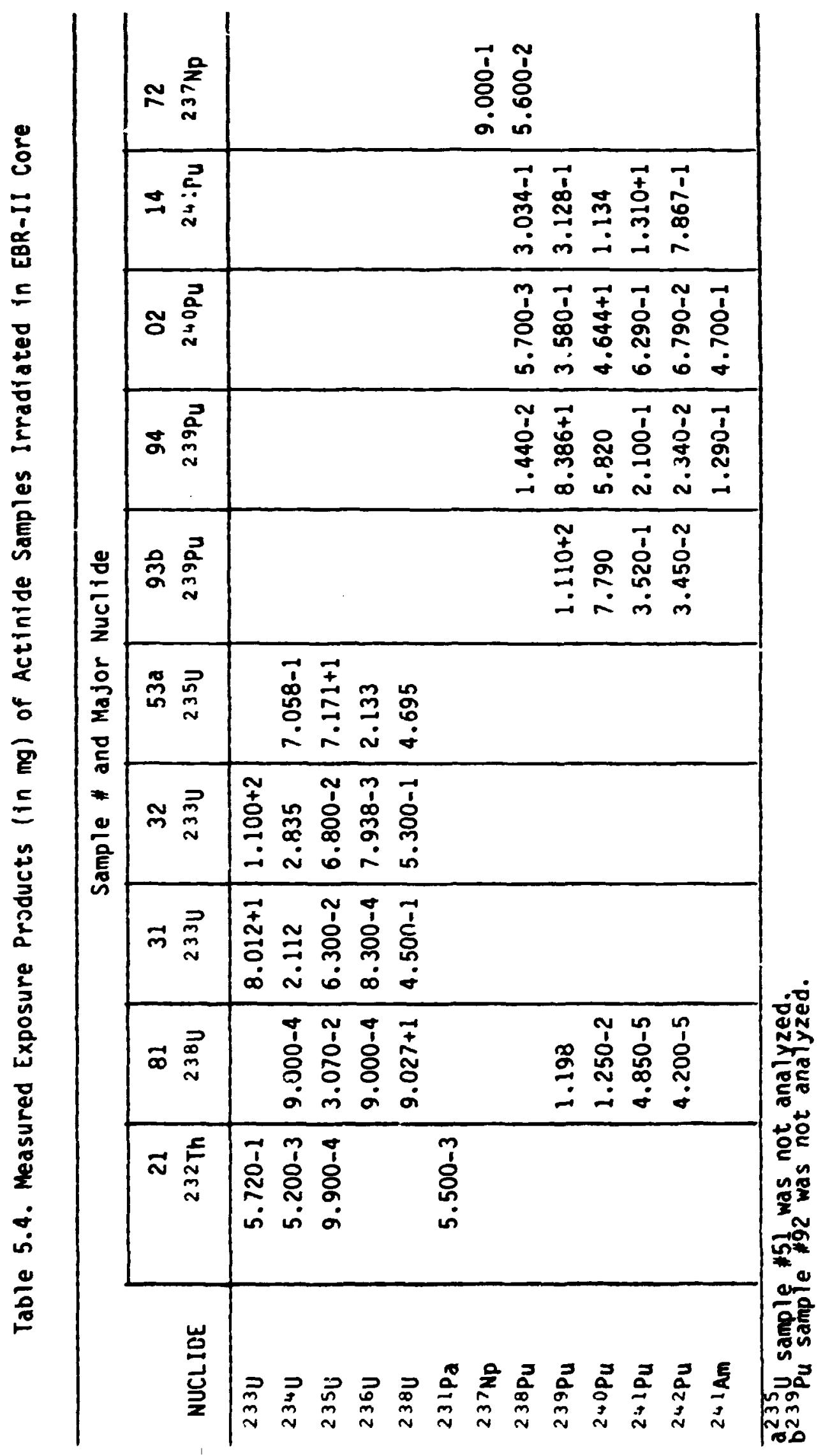


Table 5.5. Calculated Exposure Products of Actinide Samples Irradiated in EBR-Il Core (in mg)

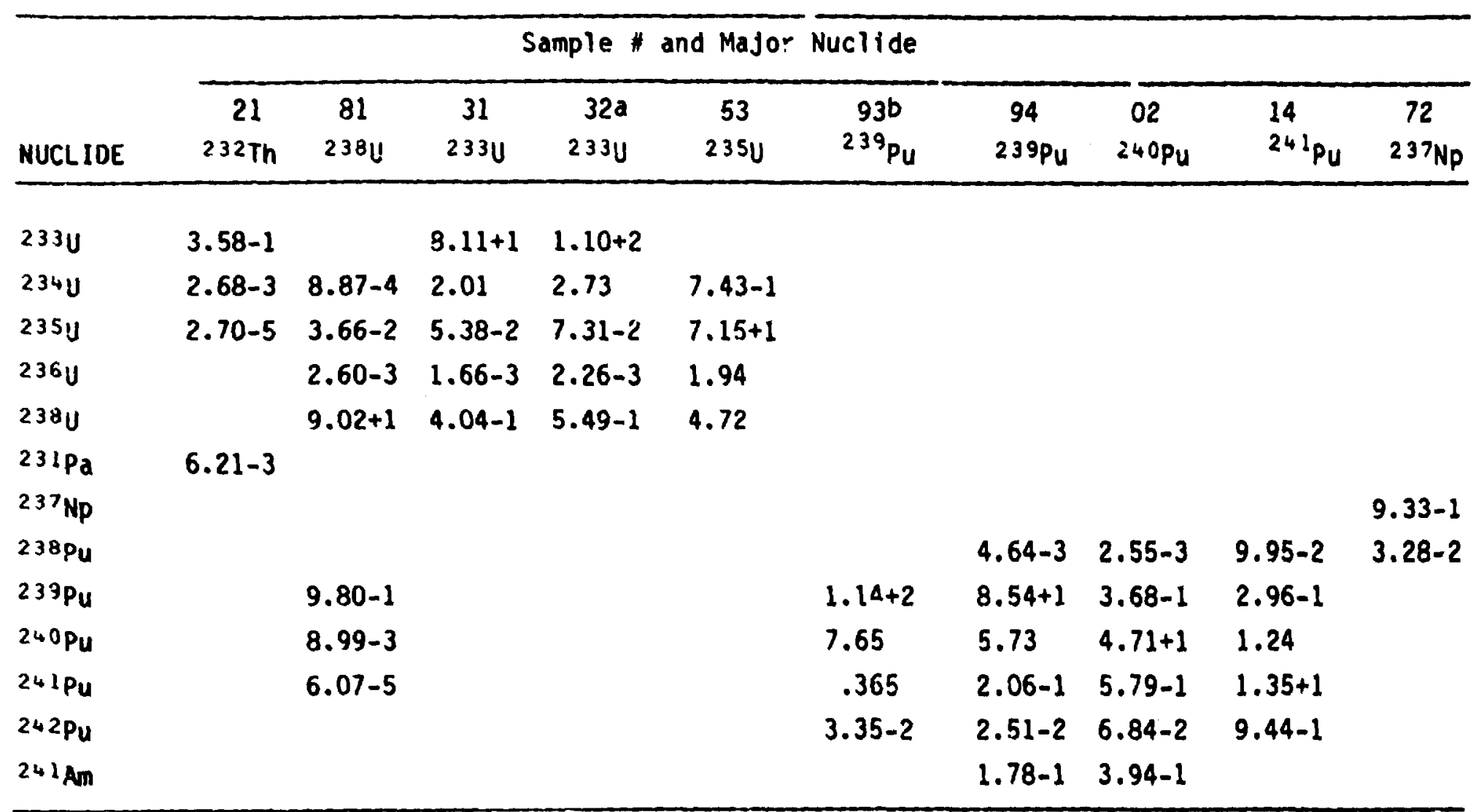

Jadjusted from sample 31 data. 
calculated end product of an isotope becomes insensitive to the input parameters if substantial amounts of this isotope were present initially. This statement is especially true for the major isotope in each sample, but may also be true for other nuclides initially present as impurities as well. In Table 5.6 we have listed the $C / E$ ratios for the end products based on Tables 5.4 and 5.5. From this table it may be easily verified that there is a high correlation between nuclides with appreciable initial contents and the closeness of their $C / E$ value to 1.0 . For this reason it appears that we should check not only the $C / E$ ratios for the end products, but also the ratios for the net change in each nuclide, namely the difference between its final and initial charge. This ratio will be a better measure for the quality of the burnup calculation, no matter what quantities were initially loaded in the reactor. The C/E ratios for these ratio quantities are listed in Table 5.7. This table actually provides the essence of the whole study and we shall devote the remainder of the section to analyze its implications.

There are two ways to analyze the results in Table 5.7. One is to examine each sample separately, the other is to examine individual nuclides, regardless of wich sample they appear in. We shall start with the first approach, and then turn to the other method.

By examining the results for the individual samples we notice that for most samples the major isotope and its first daughter can be computed with an accuracy of $90 \%$ or better. Two exceptions are the $23 \%$ Th and $237 \mathrm{~Np}$. These samples show disagreements of over $30 \%$ between 


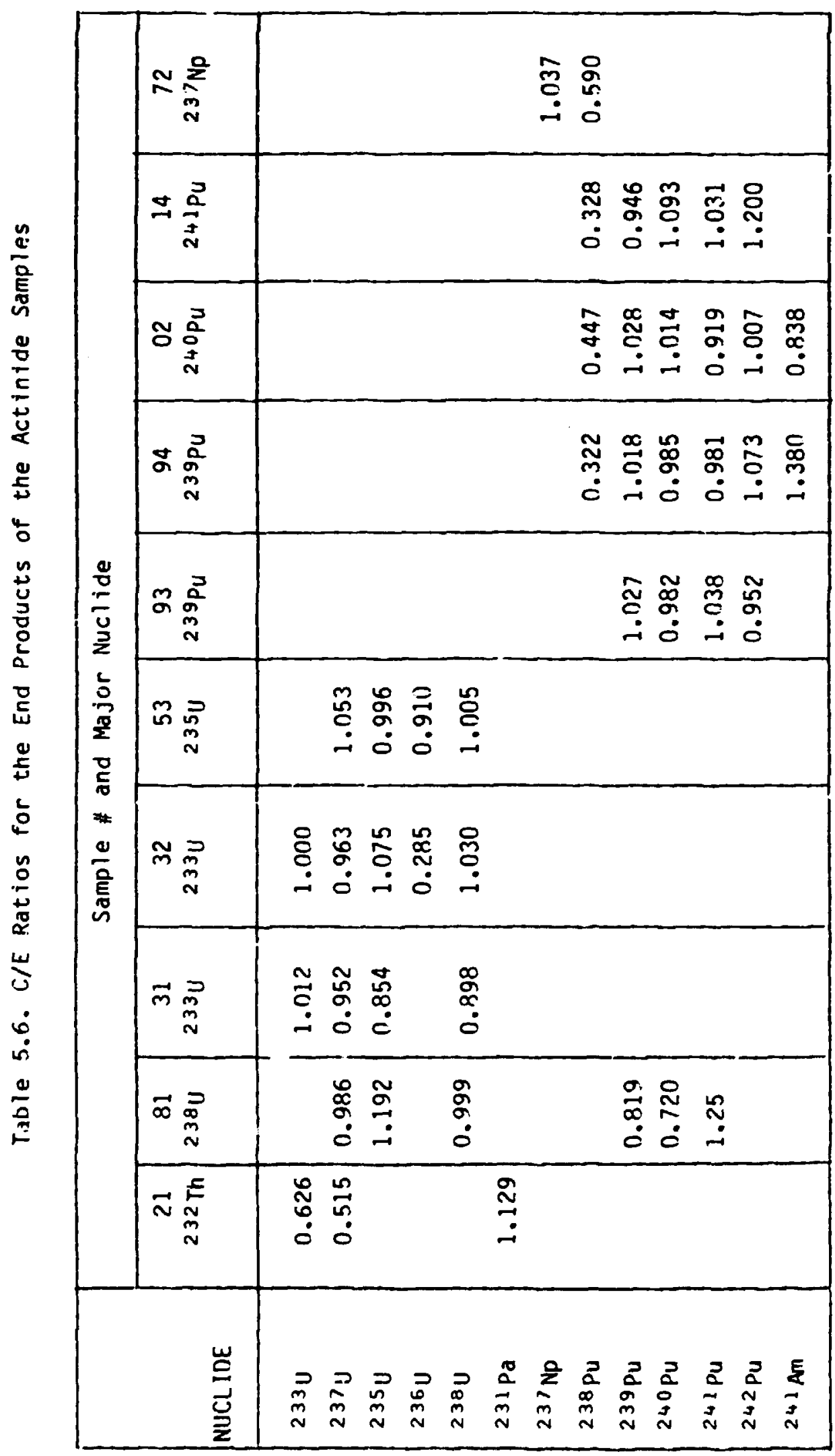




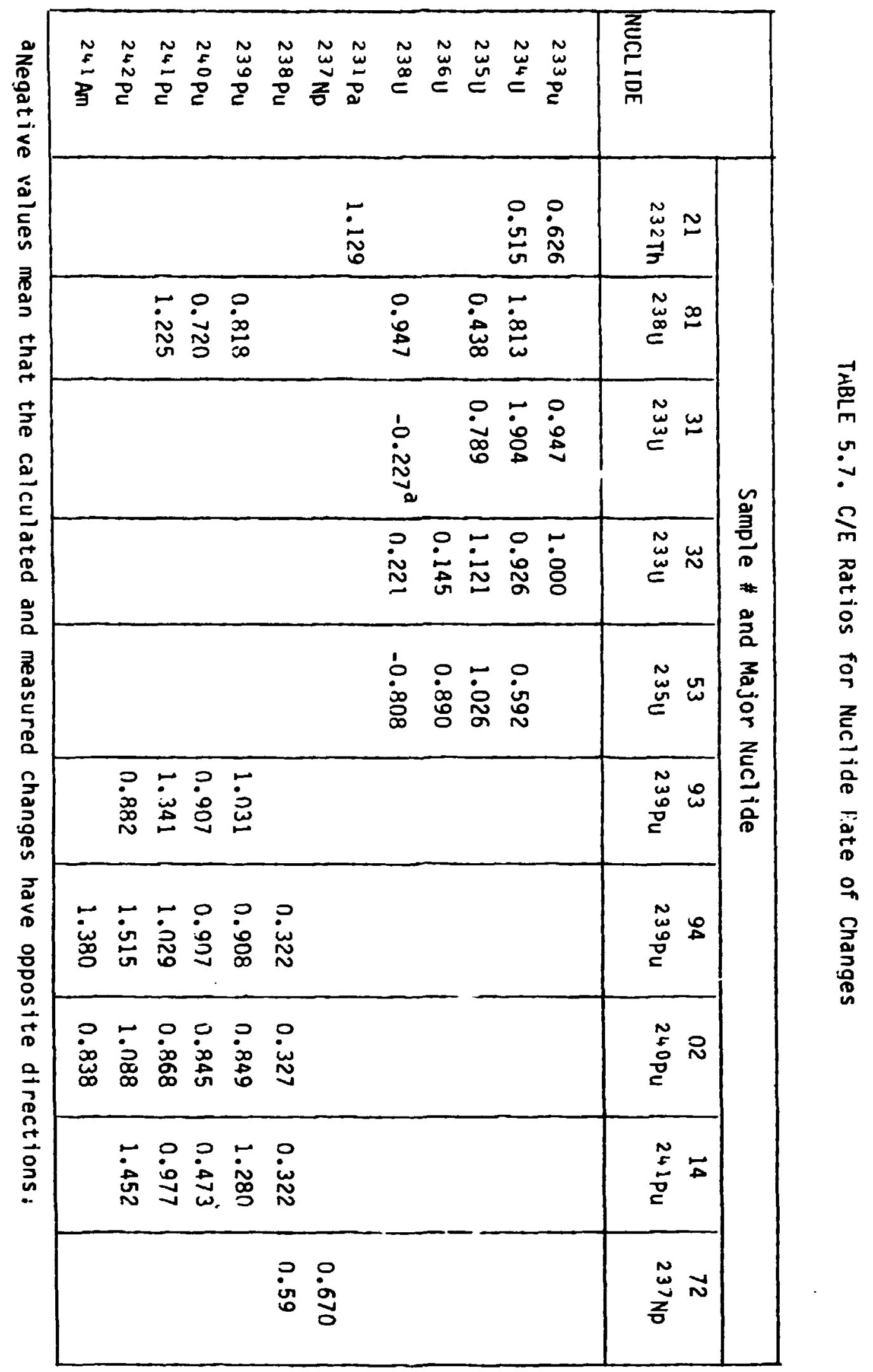


measurements and calculation. The fact that these two samples were located most closely to the core outer boundary raises the suspicion that a common reason might be responsible for the disagreement, e.g., too low flux levels or too low cross sections or both. A too low flux level may result from an underestimation of the flux drop near the core boundary, whereas too low cross sections may arise from too hard a spectrum. Whatever the reasons might he, the unsatisfactory results for these two samples definitely demonstrate the need for a well-defined experimentation in order to enable accurate calculations.

Starting with the major isotope of a sample and going up the mass chain, we notice that the major isotope in each sample and its first successor usually were calculated with similar accuracies. This is no surprise since any error in calculating the burnup of the major isotope will be directly transferred to the buildup of its first successor. Proceeding up the chain, however, we notice increasing deviation of the C/E values from 1.0. This may be attributed to two effects. First, each error made in the calculation of a nuclide is transferred to all its progeny; thus a nuclide high up the chain suffers from an accumulation of all errors made to $i$ ts precursors. The second reason is that the further up the chain, the less are the absolute amounts of the individual nuclides, so that in many cases the measured quantities of these nuclides become less accurate.

Many samples have also small amounts of impurity isotopes down the mass chain from the major isotope. Their contributions to the final products of interest is in most cases negligible, and we need not worry about their accuracy. 
The order in which the samples appear in Table 5.7 corresponds more or less to their axial arrangement in the reactor. This was done to emphasize the effect of the sample locations on the accuracy with which the exposure products may be computed. It is easily seen that the worst results are obtained for the two outermost samples, the thorium and neptunium, as already discussed above. Moving one location towards the center, we arrive at the ${ }^{238} \mathrm{U}$ and the ${ }^{241 \mathrm{Pu}}$ samples at the top and bottom, respectively. Here, the accuracy is significantly higher, but still not quite satisfactory. As a matter of fact we would like very much to be able to calculate the buildup of ${ }^{239} \mathrm{Pu}$ from $(n, y)$-capture in ${ }^{238} \mathrm{U}$ within $5 \%$, which is about the current accuracy requirements for the breeding ratio calculation in SUPER-PHENIX, 13 since this reaction has an important impact on fast breeder physics and economics. Unfortunately, the ${ }^{238} \mathrm{U}$ sample was placed at a location where the reaction rate is very sensitive to the reactor configuration, so that only moderate accuracy may be expected for this sample when our simple reactor model is used. This is also the reason for the low accuracy obtained for other peripheral samples. Arriving at the central samples, we notice a great improvement in the C/E values. As a rule for samples irradiated at locations near the core center, agreement within $10 \%$ is obtained for the major isotopes and for their first daughter, and within $20 \%$ for the next nuclide up ine chain. Results for nuclides higher up the chain are less predictable.

Now we shall look at Table 5.7 horizontally, examining individual nuclides, while varying the samples in which they occur. Starting with ${ }^{238} \mathrm{U}$, we notice that the only case where this isotope was satisfactorily measured was for the ${ }^{238} U$ sample itself. One should, however, keep in mind that $238 U$ is present in all $U$ samples because total elimination of this 
isotope is practically impossible. Small amounts of ${ }^{238} \mathrm{U}$ are usually also present in the measuring devices, which probably accounts for the observed discrepancies for this nuclide.

Difficulties in measuring ${ }^{238} \mathrm{Pu}$ may also be one reason for the su'prisingly low accuracy obtained for the ${ }^{238} \mathrm{Pu}$ in three $\mathrm{Pu}$ samples: the measured values are always much higher than the computed values. Another

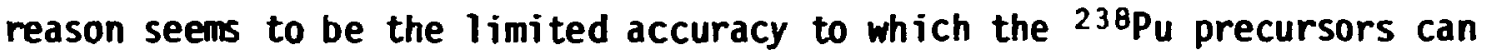
be computed. Recall that $2: 8 \mathrm{Pu}$ may be produced by two different channels: either by $(n, 2 n)$ reaction on ${ }^{239} \mathrm{Pu}$ or by a decay of ${ }^{242} \mathrm{Cm}$. The ${ }^{238} \mathrm{Pu}$ produced in the ${ }^{239} \mathrm{Pu}$ sample comes mainly from the first reaction, whereas the second channel is responsible for the buildup of ${ }^{238} \mathrm{Pu}$ in the ${ }^{241} \mathrm{Pu}$

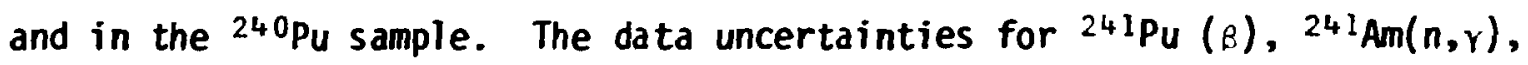
${ }^{242} \mathrm{Am}(\beta)$, and ${ }^{242} \mathrm{Cm}(\alpha)$ are large and might therefore be the primary reason for the poor $238 \mathrm{pu}$ results.

Examination of the other Pu nuclides reveals fair accuracy in most cases. It should be noted also that many calculated values are correlated - an error in the calculation for one isotope affects other isotopes as well. We shall return to this point in the next chapter.

Finally, we note that the results for ${ }^{241} \mathrm{Am}$ are fairly good, considering the fact that Am is located relatively high up the depletion chain. This result is actually a consequence of the fact that ${ }^{241}$ Am is a direct decay product of $24 \mathrm{lPu}$, which implies that the $24 \mathrm{Am}$ concentration depends mainly on the accuracy to which the $241 \mathrm{Pu}$ concentration is known.

Summarizing this section, we have found reasonable agreement between calculation and experiment for most samples located near the core center. 
As the samples approach the axial blanket, problems start to appear due to a breakdown in the accuracy of the spherical reactor model. Other C/E discrepancies (e.g., for $238 \mathrm{Pu}$ ) are probably related to errors in the experimentai measurements, especially for those nuclides whose concentrations are very small. 
54 


\section{SENSITIVITY ANALYSIS OF INPUT PARAMETERS AND THEIR IMPACT ON CALCULATED RESPONSES}

\section{A. GEMERAL REMARKS}

Table 5.7 of the previous chapter reveals some discrepancies between experimerts and calculations which are intolerably large. For example, the discrepancy between the calculated and measured amounts of $239 \mathrm{Pu}$ in the ${ }^{23 \mathrm{O}} \mathrm{U}$ sample and the ${ }^{238 \mathrm{Pu}}$ amount in all $\mathrm{Pu}$ samples indicates that some problemi exists in these analyses, possibly arising from input data. The difficulty is determining what data in the complex chain of calculations and experiments contributes most to the discrepancies between the measured and calculated results. To answer this question, sensitivity theory seems to be most appropriate.

A full sensitivity analysis of the experiments described in the previous chapters is far beyond the scope of this chapter. We shall limit the following discussion to a few selected questions only, which we find to be of general importance. Also, we seek conclusions which might be helpful in subsequent experiments, e.g., the irradiation of actinide samples in the UK Prototype Fast Reactor (PFR).

Sensitivity analyses seek to answer the following question: How much will the "response" of a system be changed if a certain input parameter is changed by an arbitrary (small) quantity? The information from sensitivity calculations can therefore be used to ascertain the input parameters which have the largest impact on the calculated results. This is usefu? in juciging whether the uncertainties in the input parameters are large enougr to account for the differences between measurements and calculations, or whether the differences can be only attributed to uncertainties in the experimental measurements. In the following, examples for both possibilities will be givell. 
The input parameters for the calculations may be categorized into three classes, according to their overall impact on the system responses, which correspond here to the exposure products. These three are:

a) initial charges and impurities, b) flux levels, and c) flux-weighted cross sections and decay constants. In the following sections these three types of parameters will be discussed, but first we shall recall the most important aspects of sensitivity analysis for depletion calculations.

The time-dependent equation for the exposure products in a sample irradiated at position $\underline{x}_{0}$ is given in Chapter IV.C and will be repeated for convenience:

$$
\frac{d \underline{N}\left(\underline{X}_{0}, t\right)}{d t}=\left[\phi(\max ) \stackrel{M}{=}\left(\sigma\left(\underline{X}_{0}\right)\right) \psi\left(\underline{X}_{0}\right) \theta(t)+\underline{\lambda}\right] N\left(\underline{X_{0}}, t\right),
$$

with the initial conditions

$$
\underline{N}\left(\underline{X}_{0}, \text { to }_{0}\right)=\underline{N_{0}} \text {. }
$$

Equations (5) and (6) may be written in a compact form, as follows:

$$
\frac{d \underline{N}}{d t}=\underline{B}(t) \underline{N}(t)+\operatorname{No\delta }\left(t-t_{0}\right) \text {. }
$$

We consider now the final concentration of a selected nuclide in a sample at $\underline{X}_{0}$ as our "system response," designated by $R$, where

$$
R=N i\left(X_{0}, t_{f}\right) \text {, }
$$

and ask for the change $\Delta R$ in this response due to a given (smal1) perturbation in one of the system input parameters appearing in $\underline{B}$ or № of (7). 
To answer this question we define the adjoint equation to (7) as:

$$
\frac{d \underline{N}^{+}}{d t}=-\underline{B}^{+}(t) \underline{N}^{+}(t)+\underline{s}^{+} \delta\left(t-t_{f}\right) \text {, }
$$

where

$$
\underline{B}^{+}=\underline{B}^{T}
$$

In our special case the adjoint source $S^{+}$(which is equivalent to a final condition) is given by:

$$
S_{j}^{+}=1 \quad \begin{aligned}
& j=i \\
& j \neq i
\end{aligned}
$$

$i$ being the index of the response nuclide of interest. The change in the reactor response due to a variation $\Delta \underline{B}$ of $\underline{B}$ may now be given, accurate to first order, by

$$
\Delta R_{B}=\int_{t_{0}}^{t_{f}} d t \underline{N}^{+}(t) \underline{\underline{B}} \underline{N}(t)
$$

and the change due to a variation in the initial condition is given exactly by

$$
\Delta R_{I}=\int_{t_{0}}^{t_{f}} d t N^{+}(t) \Delta_{0} \delta\left(t-t_{0}\right),
$$

which, because of the $\delta$ function at $t_{0}$, reduces to

$$
\Delta R_{I}=\underline{N}^{+}\left(t_{0}\right) \Delta N_{0}
$$


Each of the expressions in (11) and (13) assume that changes in data characterizing the samples have negligible effect on the flux seen by the sample, due to the small sample size. The discussion in the following sections is based on the expressions in (11) and (13).

\section{B. SENSITIVITIES TO INITIAL CONCENTRATION AND IMPURITIES}

Using Eq. (13) we define a relative sensitivity coefficient for the final amount of nuclide $i$ to the initial amount of nuclide $j$ in a sample as follows:

$$
S_{i, j}=\frac{\Delta R_{i} / R_{i}}{\Delta N_{j, 0} / N_{j, 0}}=\frac{N_{j, 0}}{R_{i}} \frac{N_{i}^{+}\left(t_{0}\right) \Delta N_{j, 0}}{\Delta N_{j, 0}}=\frac{N_{j, 0}}{R_{i}} N_{j}^{+}\left(t_{0}\right) .
$$

This coefficient expresses the percentage change of the final amount of nuclide $i$, when the initial amount of nuclide $j$ is increased by $1 \%$.

The definition of the relative sensitivity coefficient for initial concentration is a priori restricted to nuclides which are known to exist in the initial charge. However, it may happen that some unknown impurities are also included in the sample, and they too may impact the final concentrations of the nuclides. In that case, a "relative change" becomes meaningless and we must revert to the original Eq. (13), which is an exact expression for the change in the response due to a change in the vector of initial concentrations.

In Table 6.1 sensitivities to initial concentrations are given for several nuclides in selected samples. The response nuclides listed were chosen because they have unsatisfactory $C / E$ values for their burnup during irradiation. Also listed are the initial weights of the contributing nuclides. The conclusion from this table is that the major isotope of a 
sample is not necessarily the major contributor to the final quantity of interest. For instance, the production of ${ }^{241} \mathrm{Am}$ in the ${ }^{239} \mathrm{Pu}$ sample is mainly due to the presence of ${ }^{24} 1 \mathrm{Pu}$ in the initial sample, although this isotope comprises only about $0.3 \%$ of the total initial weight. A similar situation, although not as extreme, may be found for the production of $235 \mathrm{U}$ in the ${ }^{233} \mathrm{U}$ sample, where ${ }^{234} \mathrm{U}$ (about $1 \%$ of the initial weight) contributes $44 \%$ of the final $235 \mathrm{U}$ quantity. For $238 \mathrm{Pu}$ production the situation is more involved because this isotope may be produced either by $(n, 2 n)$ reactions in ${ }^{239} \mathrm{Pu}$ or by a decay of ${ }^{242} \mathrm{~cm}$. The production from the former route is usually limited because of the small $(n, 2 n)$ cross sections; therefore, the presence of any isotope which leads to the formation of ${ }^{242} \mathrm{~cm}$ may appreciably affect the $238 \mathrm{Pu}$ production. For this reason, only $68 \%$ of the $238 \mathrm{Pu}$ produced in the $239 \mathrm{Pu}$ sample is attributed to the major isotope; the rest is due to the $0.3 \%$ of ${ }^{241} \mathrm{Pu}$ in the initial charge. The fraction of the ${ }^{238} \mathrm{Pu}$ production due to $24 \mathrm{IPu}$ is even higher in the $240 \mathrm{Pu}$ sample, where $25 \%$ of the final $238 \mathrm{Pu}$ quantity originates from the initial impurity of $0.5 \% 241 \mathrm{Pu}$.

To conclude this section it should be emphasized that an accurate determination of the initial composition of all the nuclides in an irradiated sample is a primary condition for a successful evaluation of the experiment by calculational models. However, al though final amounts are usually very sensitive to the initial compositions of the samples, it seems unlikely that the discrepancies between calculations and measurements may be attributed entirely to inaccurate measurements. Unfortunately, the uncertainty of the measurements could not be verified, 
Table 6.1: Sensitivity Coefficients for Several Initial Nuclides

\begin{tabular}{|c|c|c|c|c|c|}
\hline Sample & \multicolumn{2}{|c|}{ Initial Nuclide } & $\begin{array}{l}\text { Response } \\
\text { Type }\end{array}$ & $\begin{array}{l}\text { Nuclide } \\
C / E\end{array}$ & $\begin{array}{c}\text { Sensitivity } \\
\text { Coeff. }\end{array}$ \\
\hline \multirow[t]{2}{*}{$238 \mathrm{U}(\mathbf{4 8 1 )}$} & $238 U$ & $9.129+1$ & $239 \mathrm{Pu}$ & .818 & .996 \\
\hline & $233 \mathrm{U}$ & $1.325+2$ & $235 U$ & 1.121 & .246 \\
\hline \multirow[t]{4}{*}{$233 \mathrm{U}(\# 32)$} & $234 \mathrm{U}$ & 1.413 & $235 U$ & 1.121 & .442 \\
\hline & $235 U$ & $2.553-2$ & $2351\}$ & 1.121 & .309 \\
\hline & $239 \mathrm{Pu}$ & $1.006+2$ & $238 \mathrm{Pu}$ & 0.322 & .679 \\
\hline & $241 \mathrm{Pu}$ & $3.461-1$ & $238 \mathrm{Pu}$ & 0.322 & .287 \\
\hline \multirow[t]{5}{*}{$239 \mathrm{Pu}(\# 94)$} & ${ }^{239} \mathrm{Pu}$ & $1.006+2$ & $241 \mathrm{Am}$ & 1.380 & .025 \\
\hline & $240 \mathrm{Pu}$ & 4.857 & $241 \mathrm{Am}$ & 1.380 & .190 \\
\hline & $241 \mathrm{Pu}$ & $3.461-1$ & $241 \mathrm{Am}$ & 1.380 & .770 \\
\hline & $238 \mathrm{Pu}$ & $2.660-3$ & $238 \mathrm{Pu}$ & 0.327 & .250 \\
\hline & $240 \mathrm{Pu}$ & $5.083+1$ & $238 \mathrm{Pu}$ & 0.327 & .490 \\
\hline \multirow[t]{3}{*}{$240 \mathrm{Pu}(\# 02)$} & $241 \mathrm{Pu}$ & $2.504-1$ & $238 \mathrm{Pu}$ & 0.327 & .255 \\
\hline & $240 \mathrm{Pu}$ & $5.083+1$ & $241 \mathrm{Am}$ & 0.838 & .725 \\
\hline & $241 \mathrm{Pu}$ & $2.504-1$ & $241 \mathrm{Am}$ & 0.838 & .280 \\
\hline $241 \mathrm{Pu}(\# 14)$ & $241 \mathrm{Pu}$ & $3.069+1$ & $238 \mathrm{Pu}$ & 0.322 & .987 \\
\hline
\end{tabular}


but it is believed not to exceed $5 \%$, which is not large enough to account for the observed discrepancies. This statement, however, does not exclude the possibility of existing impurities which were not measured. Such impurities might significantly contribute to the production rate of some nuclides of interest, or may unexpectedly appear in the irradiated sample as "exposure" products. Experimentalists should therefore always be aware of the eventual high importance of smail impurities which, in case they cannot be eliminated, should at least be accurately characterized.

\section{SENSITIVITIES TO CROSS SECTIONS AND FLUX LEVELS}

In the previous section we eliminated the possibility that inaccurate measurements of the initial composition of the samples could entireiy account for discrepancies between measurements and calculations. Therefore, we will examine now the impact of uncertainties of the other parameters, namely, the cross sections and flux levels on the computed results. Of particular interest are any systenatic errors which can be eliminated by adjusting various input data. In order to make this point clear, let us extract from the burnup matrix in Eq. (5) that part which is related to neutronic reactions only, by defining:

$$
\underline{B}_{N}=\phi(\max ) \underline{M}\left(\sigma\left(\underline{x}_{0}\right)\right) \omega\left(\underline{x}_{0}\right) \theta(t) .
$$

Recalling the meaning of the four terms on the right-hand side of Eq. (15), we observe that the uncertainty in $\underline{B}_{N}$ is mainly due to insufficient knowledge of the proper cross sections which enter the matrix $M$ and to 
the appropriate flux level at $\underline{x}_{0}, \phi(\max )_{\psi}\left(\underline{x}_{0}\right)$, since the time defendence of the flux normalization is thought to be accurately known. Performing the total differential of the final amounts, we may write:

$$
\Delta N i\left(t_{f}\right)=\frac{\partial N_{i}}{\partial \underline{\sigma}_{j}} \Delta \underline{\sigma}_{j}+\frac{\partial N_{i}}{\partial \phi} \Delta \phi,
$$

where $\phi$ is a short notation for $\phi i \max ) \cdot \psi\left(\underline{x}_{-}\right)$. Equation (16) is a formal expression on!y which might be replaced by a pertirsbation expression of the form (11), using the definition in (15):

$$
\frac{\partial N_{i}}{\partial \sigma_{j}} \stackrel{\Delta \sigma_{j}}{=}=\phi \int_{t_{0}}^{t_{f}} \underline{N}^{+}(t) \stackrel{\Delta \sigma_{i}}{=} \chi(t) \underline{N}(t) d t
$$

and

$$
\frac{\partial N_{i}}{\partial \phi} \Delta \phi=\Delta \phi \int_{t_{c}}^{t} \underline{N}^{+}(t) \underline{\sigma}_{i} x(t) \underline{N}(t) d t .
$$

Combining both parts of (17) we obtain:

$$
\Delta N i\left(t_{f}\right)=\int_{t_{0}}^{t_{f}} \underline{N}^{+}(t)\left[\phi \Delta \Delta_{0} i+\Delta \phi \underline{\sigma}_{i}\right] \underline{N}(t) d t .
$$

This is the basic perturbation expression for examining the effects of uncertairities in input parameters. Our approach will be based on the assımption that systematic discrepancies between measurements and calculations irise fiom possible errors in one of these parameters. 
The most common way to use Eq. (18) is to insert in its right-hand side the uncertainty values for the cross sections and the flux in order to obtain the variation of the response $\mathrm{Ni}\left(\mathrm{t}_{\mathrm{f}}\right)$. Such a procedure might enable us to decide whether the uncertainties to the input parameters are sufficient to account for the discrepancies between measurements and calculations, but it would be effective only for a single response. This is the so-called "forward sensitivity problem." In our case, however, the number of responses is large, since each nuclide in each sample acts as a response, so that a given set of uncertainties has to account for all discrepancies associated with that set. This is especially important for the flux uncertainty because $i r$ we allow a variation in $\phi$ (max), the final nuclide concentrations in all samples are expected to vary. Even if we assume a change in the flux level only at a certain location $x_{0}$ (changing the flux distribution locally), all nuclides in the sample at that location wi., obtain different final values, which may not necessarily be closer to the measured ones. For that reason we have chosen another approach known as the "inverse sensitivity problem," ".:hich will be described next. In this approach we focus first on the nuclides within a single sample and then we shall try to apply our findings to other samples. The saiple chosen was $239 \mathrm{Pu} \equiv 94$ which was located nearest to the core center and therefore is expected to yield the best resilts. However, as may be see 1 from Table 5.7 , discrepancies of $10-15 \%$ were found for the three first nuclides up the chain, even in this sample. We shall now try to analyze the source for these discrepancies. 
In the 239Pu sample analysis we concentrate on three nuclides only, assuming the discrepancies for the rest are beyond the uncertainty limits of the input parameters. These nuclides are $2{ }^{39} \mathrm{Pu}, 240 \mathrm{Pu}$ and $241 \mathrm{Pu}$, for which three equations of the form (18) may be written. In this equation we consider $\Delta N i\left(t_{f}\right)$ to be known quantities; namely, the differences between measured and calculated values, whereas the flux and cross-section variations are thought of as "variables." If there were only three unknown parameters, we could uniquely solve those three equations for the three unknowns, obtaining values for the changes to the input parameters which are required in order to exactly match the measured and calculated responses. Such a case, however, does not permit any degree of freedom for treating data uncertainties. In reality there are more than three parameters invo'ved in this chain, thus allowing a high degree of freedom in choosing appropriate changes to the input parameters. In order to determine these changes we have used an optimization technique ${ }^{14}$ based on minimization of an appropriate quadratic loss function obtained from a Bayesian inference approach. This method takes into account the uncertainties of each individual parameter and measurement. The result of this adjustment procedure is given in the following table as "best" estimates for the cross sections and flux amplitude for the $239 \mathrm{pu}$ sample. The nominal values which appear in Table 6.2 are those which were originally used to calculate the final products in the ${ }^{239} \mathrm{pu}$ sample. The uncertainty of the flux was intentionally chosen to be large in order not to constrain the results too much in advance. The remaining uncertainties were taken from Ref. 15. These, however, are uncertainties in the basic nuclear data used to obtain the one-group cross sections (ENDF/B-V) and do not 
Table 6.2 Modification of Cross Sections and Flux Level for 239 Pu Sample $\$ 94$

\begin{tabular}{|c|c|c|c|c|}
\hline Parameter & $\begin{array}{c}\text { Nominal } \\
\text { Values }\end{array}$ & $\begin{array}{c}\text { Uncertainty } \\
(\%)\end{array}$ & $\begin{array}{c}\text { Best } \\
\text { Estimate }\end{array}$ & $\begin{array}{c}\text { \% } \\
\text { Change }\end{array}$ \\
\hline $\begin{array}{c}\sigma_{\mathrm{c}}^{9^{\mathrm{a}}} \\
\sigma_{\mathrm{f}}^{9}\end{array}$ & 1.000 & 15.0 & 1.089 & 8.9 \\
$\sigma_{\mathrm{c}}^{0}$ & 1.671 & 7.0 & 0.157 & 5.4 \\
$\sigma_{\mathrm{f}}^{0}$ & .208 & 5.0 & 1.688 & 1.0 \\
$\sigma_{\mathrm{a}}^{1}$ & .713 & 10.0 & .170 & -18.3 \\
\hline
\end{tabular}

${ }^{a}$ The indices 9,0 , and 1 pertain to $239 \mathrm{Pu}, 240 \mathrm{Pu}$ and $241 \mathrm{Pu}$, respectively.

include a component arising from uncertainties in the flux spectra. Hence, the values in Table 6.2 are actually lower bounds of the uncertainties. From Table 6.2 we see that with the exception of one parameter, all changes are within the specified uncertainty limits. The only exception is for the capture cross section of $240 \mathrm{Pu}$, but even here the -hange is within two standard deviations, which is still acceptable. It should also be noted that $240 \mathrm{Pu}$ capture cross sections from ENDF/B-V are $10 \%$ higher than those from ENDF/B-IV (Table 4.8), which is larger than the other deviations. The most interesting result from Table 6.2 , however, is that the best estimate for the flux is about $9 \%$ higher than the nominal value. In fact, the flux level for each sample was never determined experimentally 
so that the nominal flux values should indeed have high uncertainties. This is also in accordance with previously detected discrepancies between power levels of EBR-II measured by different methods. 16 In Ref. 16 it was pointed out that the power level (which is proportional to the flux level) at which EBR-II was operated might be as much as $9 \pm 2 \%$ lower than a nominal value of $62.5 \mathrm{MH}$. This nominal power level is by more than $25 \%$ higher than power levels used in the present study, which are mainly based on data from Ref. 5. Therefore, it seems very reasonable to raise the flux level in our calculation by nearly $9 \%$.

In order to verify the usefulness of the suggested adjustments to the flux level and cross sections, we applied the new values to two other samples. The first was the $240 \mathrm{Pu}$ sample ( $\# 02)$ which uses the same cross sections as the ${ }^{239} \mathrm{Pu}$ sample, and the second was the ${ }^{238} \mathrm{U}$ sample (厈1) which uses different cross sections, based on the softer spectra near the core boundary. For the first sample we used the adjusted cross sections and flux level, and for the second sample the cross sections and flux level were both changed by the percentage changes given in Table 6.2. Results for these two samples compared with the original results are given in Table 6.3. This table shows improved results for most of the nuclides, with the exception of ${ }^{238} \mathrm{U}$ and ${ }^{241 \mathrm{Pu}}$. The increased disagreement for ${ }^{238} \mathrm{U}$ is caused by the increase in the flux only, since no changes were made to the uranium cross section (only Pu cross sections were modified); however, there is still an uncertainty in the ${ }^{236} \mathrm{U}$ absorption cross section which could account for the discrepancy. Also recall that a contamination problem is thought to exist for the ${ }^{238} \mathrm{U}$ measurements. The increased 
Table 6.3 Comparison Between C/E Values Obtained from Noninal and Adjusted Data

\begin{tabular}{|l|c|c|c|c|}
\hline \multirow{2}{*}{ Nuclide } & \multicolumn{2}{|c|}{$240 \mathrm{Pu}(481)$} & \multicolumn{2}{|c|}{$238 \mathrm{U}$ (芹02) } \\
\cline { 2 - 5 } $238 \mathrm{U}$ & Nominal & Best Estimate & Nominal & Best Estimate \\
\hline $238 \mathrm{Pu}$ & - & - & $1.282^{\mathrm{a}}$ & $1.588^{\mathrm{b}}$ \\
$239 \mathrm{Pu}$ & .322 & .333 & - & - \\
$240 \mathrm{Pu}$ & .849 & .939 & .818 & .885 \\
$241 \mathrm{Pu}$ & .850 & .872 & .720 & .888 \\
$242 \mathrm{Pu}$ & .881 & .739 & 1.225 & 1.349 \\
\hline
\end{tabular}

$a_{ \pm} 0.3 ; b \pm 0.37$.

disagreement for $24 \mathrm{Pu}$ in the ${ }^{240 \mathrm{Pu}}$ sample is, of course, due to the significant decrease of the $240 \mathrm{Pu}$ capture cross section, which shows that the required change in this cross section was probably overestimated by the optimization program.

It was not intended in this section to develop a completely consistent set of adjusted cross sections in order to re-evaluate the calculated exposure products; it was merely meant to reveal systematic discrepancies. As a result of the study we feel that there is a strong incentive to increase the assumed flux level by nearly 10\%. The results for other samples that have not been mentioned in this section also show that better $C / E$ values may be obtained for the major nuclide and its first processor by merely increasing the flux level by $5-10 \%$, whereas further cross-section adjustments mily probably be required in order to redice the C/E deviations 
of other nuclides from 1.0 .

The cross-section uncertainties given in Table 6.2 arise oniy from basic data uncertainties. Other uncertainties arise from the flux-weighting spectrum used in collapsing the data, especially near the core-blanket interface since the change in blanket composition was not accounted for in the calculations. To estimate this effect, one-group cross sections from Tabies 4.3 and 4.4 , which were derived from flux caiculations using two different EBR-TI models, are compared in Table 6.4. These models assumed a depleted uranium blanket and a stainless steel blanket, respectively, and the cross sections that are compared are taken from regions near the core boundary where their sensitivities to blanket composition are at a maximum. The cross sections of the first column are regarded as reference cross sections in order to define the relative changes between the different data. The changes in the collapsed data can be viewed as an upper bound to the uncertainty arising from the flux spectrum, since the two limiting cases have been considered at the location of maximum sensitivity. In the sense that this table bounds the uncertainties in one-group data, practically all cross sections have "uncertainties" which are high enough to account for most discrepancies between the measured and calculated values. It should, however, be noted that these estimated uncertainties are probably unrealistically high and also are correlated since each cross-section set in Table 6.2 was derived from a common model; i.e., if it is assumed that a certain cross section should be changed due to spectra adjustments, the whcle set of cross sections should be changed simultaneously. 
Table 6.4 The Range of Variation of One-Group Capture Cross Sections Due to Different Heighting Spectra

\begin{tabular}{|c|c|c|c|c|c|}
\hline Nuclide & $\begin{array}{l}\text { Table } 4.3 \\
\text { Region } 3\end{array}$ & $\begin{array}{l}\text { Table } 4.4 \\
\text { Region } 3\end{array}$ & $\begin{array}{c}\text { Relative } \\
\text { Change }\left(\begin{array}{l}\alpha \\
0\end{array}\right)\end{array}$ & $\begin{array}{l}\text { Table } 4.4 \\
\text { Region } 3^{-}\end{array}$ & $\begin{array}{c}\text { Relative } \\
\text { Change }\left(\begin{array}{l}\alpha \\
\vdots\end{array}\right)\end{array}$ \\
\hline${ }^{2}{ }^{3} \mathrm{U}$ & .1570 & .1763 & 12.3 & .2264 & 44.2 \\
\hline${ }^{234} \mathrm{U}$ & .3286 & .4366 & 32.9 & .6887 & 109.6 \\
\hline $235 U$ & .2716 & .2938 & 8.2 & .3676 & 35.3 \\
\hline${ }^{238} \mathrm{U}$ & .1501 & .1569 & 4.5 & $\therefore 843$ & 22.8 \\
\hline $23 \ni P u$ & .1895 & .2426 & 28.0 & .3831 & 102.2 \\
\hline $240 \mathrm{Pu}$ & .2505 & .4453 & 77.8 & 1.0107 & 303.5 \\
\hline $24 \mathrm{IPU}$ & .2291 & .2718 & 18.6 & .3791 & 65.5 \\
\hline
\end{tabular}

We have not considered here the uncertainties of the fission cross sections. The sensitivity of the results to these data are usually a factor of 2 lower than for the capture cross sections. They significantly affect only the disappearance of the major nuclides, while their importance for the transformation process is, in general, negligible. 
$-10$ 
VII. SUMAARY AND CONCLUSIONS

This report presents a comparison of experimental versus calculated results for the burnup of actinide samples irradiated in EBR II during 1967-1970. The experimental analysis consisted of mass spectrometer and alpha counting measurements to determine the exposure products in the irradiated samples. The calculational analysis employed a spherical model of the EBR configuration to obtain spacedependent flux spectra for multi-group averaging of the latest ENDF/B-V datd. The spatial shape of the flux distribution was obtained from earlier ANL reports. Burnup calculations were performed for each sample with the ORIGEN code.

Table 7.1 presents an overall summary of our results. In many cases the agreement between the measured and calculated postirradiation composition of the samples is quite satisfactory. The differences between the measured and calculated values can be attributed to at least three different possibilities: (a) the values taken for the cross sactions, (b) the assessment of the total neutron flux and its energy composition, and (c) characterization of the initial isotopic composition of the samples, especially those nuclides with low abundance.

These several possibilities are illustrated by the results for ${ }^{238} \mathrm{Pu}$ observed in the irradiated ${ }^{239} \mathrm{Pu}$ (\#94) sample. The observed amount of ${ }^{238} \mathrm{Pu}$ is almost 3 times larger than the calculated anount. One possibility for this difference is that the amount of ${ }^{2.38} \mathrm{Pu}$ in the original sample is not known (we assumed it was zero). Secondly, the 
Table 7.1 Summary of Experimental and Calculated Isotopic Composition of Irradiated Actinide Samples

\begin{tabular}{|c|c|c|c|c|}
\hline Sample & Muclide & $\begin{array}{l}\text { Experinental } \\
\text { Initial } \\
\text { Weight } \\
\text { (min) }\end{array}$ & $\begin{array}{l}\text { Experinental } \\
\text { Final } \\
\text { Weight } \\
\text { (mg) }\end{array}$ & $\begin{array}{l}\text { Calculated } \\
\text { Final } \\
\text { Weight } \\
\text { (no) }\end{array}$ \\
\hline $\operatorname{Th} 232$ (\$21) & $\begin{array}{r}\text { in232 } \\
\text { U233 } \\
\text { U234 } \\
\text { U235 } \\
\text { Pa231 }\end{array}$ & 32.81 & $\begin{array}{l}0.57 \\
0.005 \\
0.001 \\
0.005\end{array}$ & $\begin{array}{c}0.36 \\
0.003 \\
<.0001 \\
0.006\end{array}$ \\
\hline U238 (\$81) & $\begin{array}{l}\text { U234 } \\
\text { U235 } \\
\text { U236 } \\
\text { U236 } \\
\text { Pu239 } \\
\text { Pu24n } \\
\text { Pu241 } \\
\text { Pu242 }\end{array}$ & $\begin{array}{l}n .0009 \\
0.041 \\
0.9017 \\
91.54\end{array}$ & $\begin{array}{l}n .0009 \\
n .031 \\
0.0009 \\
0 n .27 \\
1.20 \\
0.013 \\
9.000555 \\
n .00 M 4\end{array}$ & $\begin{array}{l}0.0009 \\
0.037 \\
0.0026 \\
90.2 \\
0.98 \\
0.009 \\
0.00006\end{array}$ \\
\hline $0233(-31)$ & $\begin{array}{l}\text { U233 } \\
\text { U234 } \\
\text { U235 } \\
\text { U236 } \\
\text { U238 }\end{array}$ & $\begin{array}{c}99.6 \\
1 . n 5 \\
0.019 \\
\times n .091 \\
0.41\end{array}$ & $\begin{array}{c}80.1 \\
2.11 \\
0.063 \\
0.001 \\
0.45\end{array}$ & $\begin{array}{l}81.1 \\
2.01 \\
0.054 \\
0.002 \\
0.4 r\end{array}$ \\
\hline U235 (.53) & $\begin{array}{l}\text { U234 } \\
\text { U275 } \\
\text { U236 } \\
\text { U238 }\end{array}$ & $\begin{array}{c}0.81 \\
87.2 \\
0.39 \\
4.37\end{array}$ & $\begin{array}{c}0.71 \\
71.7 \\
2.13 \\
4.70\end{array}$ & $\begin{array}{r}0.74 \\
71.5 \\
1.94 \\
4.72\end{array}$ \\
\hline Pu239 (\$33) & $\begin{array}{l}\text { Pu239 } \\
\text { Pu240 } \\
\text { Pu241 } \\
\text { Pu242 }\end{array}$ & $\begin{array}{c}130.3 \\
6.29 \\
0.45 \\
0.026\end{array}$ & $\begin{array}{c}111.0 \\
7.79 \\
0.35 \\
0.934\end{array}$ & $\begin{array}{c}114.0 \\
7.65 \\
0.37 \\
0.034\end{array}$ \\
\hline Pu233 (:94) & $\begin{array}{l}\text { Pu239 } \\
\text { Pu239 } \\
\text { Pu24C } \\
\text { Pu241 } \\
\text { Pu24? } \\
\text { An24i }\end{array}$ & $\begin{array}{l}100.6 \\
4.86 \\
0.35 \\
0.020\end{array}$ & $\begin{array}{l}0.014 \\
83.9 \\
5.82 \\
0.21 \\
0.023 \\
0.13\end{array}$ & $\begin{array}{c}0.005 \\
85.4 \\
5.73 \\
0.21 \\
0.025 \\
0.18\end{array}$ \\
\hline $4233(132)$ & $\begin{array}{l}\text { U233 } \\
\text { U234 } \\
\text { U235 } \\
\text { U236 } \\
\text { U238 }\end{array}$ & $\begin{array}{c}132.5 \\
1.41 \\
0.026 \\
<0.001 \\
0.55\end{array}$ & $\begin{array}{c}110.0 \\
2.84 \\
0.068 \\
0.007 \\
0.53\end{array}$ & $\begin{array}{c}110.9 \\
2.73 \\
0.073 \\
0.002 \\
0.55\end{array}$ \\
\hline Pu240 $(114)$ & $\begin{array}{l}\text { Pu278 } \\
\text { Pu239 } \\
\text { Pu240 } \\
\text { Pu241 } \\
\text { Pu242 } \\
\text { Am241 }\end{array}$ & $\begin{array}{c}0.001 \\
0.42 \\
50.8 \\
0.25 \\
0.062\end{array}$ & $\begin{array}{c}n .076 \\
0.36 \\
46.4 \\
0.63 \\
0.068 \\
0.47\end{array}$ & $\begin{array}{l}0.003 \\
0.37 \\
47.1 \\
0.58 \\
0.068 \\
0.30\end{array}$ \\
\hline Pu24) ( 02$)$ & $\begin{array}{l}\text { Pu238 } \\
\text { Pu239 } \\
\text { Pu24n } \\
\text { Pu241 } \\
\text { Pu24? }\end{array}$ & $\begin{array}{c}0 . n 03 \\
0.37 \\
1.34 \\
30.7 \\
0.44\end{array}$ & $\begin{array}{c}0.3 n \\
0.31 \\
1.13 \\
1.13 \\
0.79\end{array}$ & $\begin{array}{c}0.10 \\
0.30 \\
1.24 \\
13.5 \\
7.94\end{array}$ \\
\hline Np237 (+7?) & $\begin{array}{l}\text { Np237 } \\
\text { Pu238 }\end{array}$ & $1.0 n$ & $\begin{array}{l}0.90 \\
0.06\end{array}$ & $\begin{array}{l}0.93 \\
0.03\end{array}$ \\
\hline
\end{tabular}


important process for the production of ${ }^{238} \mathrm{Pu}$ in the ${ }^{239} \mathrm{Pu}$ sample is the $(n, 2 n)$ process. This process depends on the flux of high energy neutrons. As illustrated in Table 5.1, the relative amount of flux in the region above $2 \mathrm{MeV}$ differs by 50 percent in the ANL and ORNL flux spectra. The ANL flux spectra would have predicted even less ${ }^{238} \mathrm{Pu}$. In this particular case of ${ }^{238} \mathrm{Pu}$ production in the ${ }^{239} \mathrm{Pu}$ sample, we strongly suspect that the observed difference is caused by assuming cross-section values for the ${ }^{239} \mathrm{Pu}(n, 2 \mathrm{n})$ process which are too small. This cross section has not been measured; it was drawn in from general systematics.

It was found that the spherical reactor model gave reasonable results near the core center but was inadequate near the blanket regions. It is estimated that an uncertainty of around $20 \%$ should be assigned to the calculated results due to a combination of methods and data uncertainties. Sensitivity analysis indicates that small amounts of impurities in the samples can strongly influence the measured results for those materials wich are produced in minute quantities, and therefore the initial sample composition is another source of uncertainty.

It is disappointing that this large an uncertainty must be assigned to the results, but this fact illustrates the importance of having a well planned and documented experimental program in which preanal; sis calculations play an important role. The present EBR II experiment was somewhat lacking in this regard because the original 
experiment was designed by ANL for other purposes, and it was then difficult for ORNL to resurrect all the necessary information after ten years had elapsed.

A comparison was made of the 1-group effective EBR cross sections based ENDF/B-IV and $V$ data. Averaged over the hard EBR-II spectrum at the core center, the cross sections for ${ }^{235} U$ fission, ${ }^{238} U$ capture, and ${ }^{239} \mathrm{Pu}$ changed by $-0.9,2.3$, and $-0.77 \%$, respectively, going from ENDF/B-IV to V.

The buildup of most transmutation products in the $\mathrm{Pu}$ samples was computed to within $15 \%$ using ENDF/B-V data. The production of ${ }^{239} \mathrm{Pu}$ in the ${ }^{239} \mathrm{U}$ sample was underestimated by $20 \%$, possibly due to the close proximity of this sample to the axial blanket where the flux spectrum was poorly represented. A simple least-squares adjustment of the input data to the burnup calculation was performed on the ${ }^{239} \mathrm{Pu}$ sample to reduce the $C / E$ values. A systematic adjustment of $5-10 \%$ in the EBR flux normalization was indicated, which could imply that earlier EBR measurements underestimated the power level. Unfortunately, no reliable information could be obtained on the buildup of americium and curium due to large experinental uncertainties in measuring the small quantities of these materials. The future PFR experiment should provide much better data on the higher actinide cross sections.

As noted in Section I, a primary goal of the present study was to gain experience for a future irradiation study to be performed in the United Kingdom's PFR. Several important lessons have been learned from problems encountered in the EBR-II analysis, which are to be corrected for the PFR program. These are the following: 
(a) Results are sensitive to small amounts of impurities in the initial actinide specimens. The PFR samples should be made as pure as possible, and any isotopic impurities should be accurately characterized using mass spectometry.

(b) Heavy metal impurities in the Ni capsule material influenced the mass spectrometer measurements. A capsule made of a material such as high purity vanadium should be used in the PFR experiment.

(c) Experiment analysis must be done in a very clean environment to avoid contamination from other experiments. It is recommended that new glove boxes be used for PFR analysis.

(d) The effect of capsule perturbation on the flux should be examined and corrected for.

(e) The effect of thermal reactions on the sample burnup should be identified and corrected for.

(f) Accurate documentation of reactor operating history and power level is imperative; a reasonable estimate for the uncertainty in the absolute reactor power level (flux normalization) should be obtained.

(g) The axial and radial flux shapes should be obtained with 3-0 diffusion theory; any time dependence in the relative shapes and spectra should be identified.

(h) Dosimeters should be strategically placed to aid in determining flux spectra and shape. It is recommended that a least squares adjustment be done on the 3-D diffusion theory calculations to minimize differences between measured and computed dosimeter values. The 
resulting flux spectra, axial and radial shape factors, and normalization should then be used as in the present EBR-II analysis.

(i) Time-dependent sensitivity analysis was shown in the present study to be a useful tool for understanding discrepancies. It is recommended that a similar type of analysis be performed for the PFR experinent. 


\section{REFERENTES}

1. J. T. Madell, R. E. Jarka, "Analysis of Experimental Subassemblies in EBR-II," Argonne National Laboratory, ANL-7410 (1969).

2. R. J. Meyer, R. P. Larsen, "Experiment Description and Hazards Evaluation for ANL-MET-CEN Irradiation Capsule for EBR-II Subassembly X018," Argonne National Laboratory, ANL-RCV-4084, (1966).

3. H. A. Larson, P. Kalker, D. Meneghetti, "EBR-II Burnup and Fluence History I," Part 2, Argonne National Laboratory, ANL/ECRO-085 (1974).

4. D. Meneghetti, R. H. Rempert, "Calculational Analysis of Tests of EBR-II Capture-to-Fission Ratios," Argonne National Laboratory, ANL-7942 (1972).

5. J. C. Case, "Core Loading Diagram for ZBR-II Runs 4 Through 38, April 1965-Mid-December 1969," Ar: unne National Laboratory, ANL/EBR-7 (1969).

6. D. H. Smith, ed., "Mass Spectrometry in the Analytical Chemistry Division of ORNL," ORNL/TM-6485.

7. R. !. Walker, R. E. Eby, C. A. Pritchard, J. A. Carter, "Simultanejus Plutonium and Uranium Isotope Analysis from a Single Resin Bed - A Simplified Chemical Technique for Assaying Spent Reactor Fuels," Analytical Letters (B \& 9), 563-574 (1974).

8. D. A. Kucera, J. T. Made11, "Analysis and Development of Nectron Cross Sections for EBR-II Studies," Argonne National Laboratory, ANL-7410 (1969).

9. N. M. Greene, W. E. Ford, III, J. L. Lucius, J. E. White, L. M. Petrie, R. Q. Wright, "AMPX: A Modular Code System for Generating Coupled Multigroup Neutron-Gamma Libraries from ENDF/B," ORNL/TM-3706, ai so PSR-6.3.

10. M. J. Bell, "ORIGEN-The ORNL Isotope Generation and Depletion Code," ORNL-6428. (1973).

11. D. Meneghetti, E. R. Eber 301 , P. Walker, "Analysis of Burnups in EBR-II Oriver Fuel Elements," Nuclear Technology, Vol. 25, (Feb. 1975). 
12. R. R. Heinrich, J. Milliaams, A. A. Madson, N. D. Dudly, "Integral Measurements in EBR-II of Capture Rates, Fission Rates and Alpha for $233 \mathrm{U}, 235 \mathrm{U}, 238 \mathrm{U}, 239 \mathrm{Pu}, 240 \mathrm{Pu}$, and 242Pu," Argonne National Laboratory, ANL-7791 (1971).

13. P. Hammer, in "Proceedings of the Conference: 1980 Advances in Reactor Physics and Shielding," p. $450 \mathrm{ff}$., Sun Valley, Idaho, September 14-29, 1980.

14. J. Barhen, J. J. Nagschal, "NOGA, A Nonlinear Optimization and Generalized Adjustments Code for Uncertainty Analysis of Transient Systems," ORML/TM-7544 (1981).

15. M. L. Hillians, "Perturbation and Sensitivity Theory for Reactor Burnup Analysis," ORNL/TM 7096 (1979).

16. G. H. Golden et al., "Correlation and Interpretation of Data Relative to EBR-II Power Level" in Proc. Am. Nucl. Soc. Topical Meeting on Irradiation Experimentation in Fast Reactors, väckson Lake Lodge, WY (Ept. 197.7). 
APPENDIX A

IRRADIATION HISTORY OF THE ACTINIDE SAMPLES IN EBR-II

\begin{tabular}{|c|c|c|c|c|c|c|c|}
\hline Run No. & $\begin{array}{c}\text { Date } \\
\text { Started } \\
\end{array}$ & $\begin{array}{r}\text { Date } \\
\text { Ended }\end{array}$ & $\begin{array}{l}\text { Oper. } \\
\text { Days }\end{array}$ & $\begin{array}{r}\text { Total } \\
\text { Energy } \\
\text { Output } \\
\text { (MNd) } \\
\end{array}$ & $\begin{array}{l}\text { Avg. } \\
\text { Power } \\
\text { (MW) }\end{array}$ & $\begin{array}{l}\text { Samples } \\
\text { Location }\end{array}$ & $\begin{array}{l}\text { Shut- } \\
\text { down } \\
\text { Period } \\
\text { (Days) }\end{array}$ \\
\hline 24 & $12 / 6 / 66$ & $12 / 31 / 66$ & 25 & 630 & 25.20 & Row 2 & 107 \\
\hline $25 A$ & $4 / 17 / 67$ & $6 / 19 / 57$ & 63 & 641 & 10.17 & " 2 & 2 \\
\hline $25 B$ & $6 / 21 / 67$ & $6 / 27 / 67$ & 6 & 150 & 25.00 & $" 2$ & 2 \\
\hline $25 c$ & $6 / 29 / 67$ & $7 / 20 / 67$ & 21 & 723 & 34.13 & " 2 & 1 \\
\hline 250 & $7 / 21 / 67$ & $1 / 22 / 67$ & 1 & 34 & 34.00 & Out & 1 \\
\hline $25 E$ & $7 / 23 / 67$ & $8 / 18 / 67$ & 26 & 13 & 0.50 & Row 2 & 35 \\
\hline $26 \mathrm{~A}$ & $9 / 22 / 67$ & $9 / 29 / 67$ & 7 & 87 & 12.43 & " 2 & 12 \\
\hline $26 B$ & $10 / 11 / 67$ & $11 / 20 / 67$ & 40 & 1029 & 25.73 & " 2 & 2 \\
\hline $26 C$ & $11 / 22 / 67$ & $12 / 12 / 67$ & 21 & 619 & 29.48 & " 2 & 52 \\
\hline $27 A$ & $2 / 2 / 68$ & $2 / 29 / 68$ & 27 & 283 & 10.48 & $" 2$ & 3 \\
\hline $27 B$ & $3 / 1 / 68$ & $3 / 5 / 68$ & 4 & 177 & 44.25 & $" 2$ & 2 \\
\hline $27 C$ & $3 / 7 / 68$ & $3 / 11 / 68$ & 4 & 62 & 15.50 & $" 2$ & 19 \\
\hline 270 & $3 / 30 / 68$ & $4 / 5 / 68$ & 7 & 286 & 40.86 & $" 2$ & 1 \\
\hline $27 E$ & $4 / 7 / 68$ & $4 / 11 / 68$ & 4 & 90 & 22.50 & " 2 & 2 \\
\hline $27 \mathrm{~F}$ & $4 / 13 / 68$ & $4 / 16 / 68$ & 3 & 68 & 22.67 & " 2 & 1 \\
\hline $27 G$ & $4 / 17 / 68$ & $4 / 19 / 68$ & 2 & 49 & 24.50 & " 2 & 6 \\
\hline $27 \mathrm{H}$ & $4 / 25 / 68$ & $5 / 2 / 68$ & 7 & 206 & 29.43 & " 2 & 1 \\
\hline $27 I$ & $5 / 3 / 68$ & $5 / 6 / 68$ & 3 & 96 & 32.00 & $" 2$ & 3 \\
\hline $28 \mathrm{~A}$ & $5 / 9 ; 68$ & $5 / 13 / 68$ & 4 & 154 & 38.50 & " 2 & 2 \\
\hline $28 B$ & $5 / 15 / 68$ & $5 / 27 / 68$ & 12 & 303 & 25.25 & " 2 & 2 \\
\hline $28 \mathrm{C}$ & $5 / 29 / 68$ & $6 / 15 / 68$ & 17 & 669 & 39.35 & $" 2$ & 11 \\
\hline
\end{tabular}




\begin{tabular}{|c|c|c|c|c|c|c|c|c|}
\hline Run No. & $\begin{array}{c}\text { Date } \\
\text { Started } \\
\end{array}$ & $\begin{array}{r}\text { Date } \\
\text { Ended } \\
\end{array}$ & $\begin{array}{l}\text { Oper. } \\
\text { Days }\end{array}$ & $\begin{array}{r}\text { Total } \\
\text { Energy } \\
\text { Output } \\
\text { (mid) } \\
\end{array}$ & $\begin{array}{r}\text { Avg. } \\
\text { Power } \\
\text { (Mu) } \\
\end{array}$ & $\begin{array}{l}\text { Sampl } \\
\text { Locat } \\
\end{array}$ & $\begin{array}{l}\text { les } \\
\text { tion } \\
\end{array}$ & $\begin{array}{l}\text { Shut- } \\
\text { down } \\
\text { Period } \\
\text { (Days) }\end{array}$ \\
\hline $29 A$ & $6 / 26 / 68$ & $7 / 5 / 68$ & 9 & 188 & 20.89 & Row & 2 & 4 \\
\hline $29 B$ & $7 / 9 / 68$ & $7 / 11 / 68$ & 2 & 24 & 12.00 & " & 2 & 4 \\
\hline $29 C$ & $7 / 15 / 68$ & $7 / 22 / 68$ & 7 & 205 & 25.29 & " & 2 & 3 \\
\hline 290 & $7 / 25 / 68$ & $8 / 16 / 78$ & 22 & 710 & 32.21 & 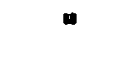 & 2 & 5 \\
\hline $30 A$ & $8 / 21 / 68$ & $9 / 5 / 68$ & 15 & 652 & 43.47 & " & 2 & 2 \\
\hline $30 \mathrm{~B}$ & $9 / 7 / 68$ & $9 / 11 / 68$ & 4 & 166 & 41.50 & $n$ & 2 & 0 \\
\hline $30 \mathrm{C}$ & $9 / 11 / 68$ & $9 / 15 / 68$ & 4 & 52 & 13.00 & $"$ & 2 & 2 \\
\hline 300 & $9 / 17 / 68$ & $9 / 21 / 68$ & 4 & 79 & 19.75 & & 2 & 1 \\
\hline $30 E$ & $9 / 22 / 68$ & $9 / 25 / 68$ & 3 & 79 & 26.33 & $"$ & 2 & 5 \\
\hline $31 \mathrm{~A}$ & $9 / 30 / 68$ & $10 / 4 / 68$ & 4 & 89 & 22.25 & $"$ & 2 & 7 \\
\hline $31 \mathrm{~B}$ & $10 / 11 / 68$ & $10 / 18 / 68$ & 7 & 15.9 & 22.71 & " & 2 & 1 \\
\hline $31 C$ & $10 / 19 / 68$ & $10 / 22 / 68$ & 3 & 84 & 23.00 & " & 2 & 4 \\
\hline 310 & $10 / 26 / 68$ & $11 / 3 / 68$ & 8 & 372 & 46.50 & $"$ & 2 & 1 \\
\hline $31 E$ & $11 / 4 / 68$ & $11 / 4 / 68$ & - & - & - & Dut & & 1 \\
\hline $31 F$ & $11 / 5 / 68$ & $11 / 14 / 68$ & 9 & 413 & 45.89 & $"$ & & 1 \\
\hline $31 G$ & $11 / 15 / 68$ & $11 / 17 / 68$ & 2 & 111 & 55.50 & Row & 2 & 10 \\
\hline $32 A$ & $11 / 27 / 68$ & $12 / 17 / 68$ & 20 & 852 & 42.60 & $"$ & 2 & 1 \\
\hline $32 B$ & $12 / 18 / 68$ & $12 / 24 / 68$ & 6 & 249 & 41.50 & $"$ & 2 & 1 \\
\hline $32 C$ & $12 / 25 / 68$ & $12 / 27 / 68$ & 2 & 72 & 36.00 & $"$ & 2 & 1 \\
\hline 320 & $12 / 28 / 68$ & $1 / 2 / 69$ & 5 & 224 & 44.8 & & 2 & 54 \\
\hline $33 A$ & $2 / 25 / 69$ & $3 / 29 / 69$ & 32 & 6.70 & 18.75 & out & & 9 \\
\hline $33 B$ & $4 / 7 / 69$ & $4 / 22 . / 69$ & 15 & $57 t$ & 38.40 & $"$ & & 3 \\
\hline $34 A$ & $4 / 25 / 69$ & $5 / 13 / 69$ & 18 & 822 & 45.67 & Row & 4 & 1 \\
\hline
\end{tabular}




\begin{tabular}{|c|c|c|c|c|c|c|c|c|c|}
\hline \multirow{2}{*}{ Run } & \multirow{2}{*}{$\frac{\text { No. }}{348}$} & \multirow{2}{*}{$\frac{\begin{array}{c}\text { Date } \\
\text { Started }\end{array}}{5 / 14 / 69}$} & \multicolumn{2}{|c|}{$\begin{array}{l}\text { Oper. } \\
\text { Days } \\
\end{array}$} & \multirow{2}{*}{$\begin{array}{r}\begin{array}{r}\text { Total } \\
\text { Energy } \\
\text { Output } \\
\text { (Mud) }\end{array} \\
310\end{array}$} & \multirow{2}{*}{$\begin{array}{r}\text { Avg. } \\
\text { Power } \\
\text { (MN) }\end{array}$} & \multicolumn{2}{|c|}{$\begin{array}{l}\text { Samples } \\
\text { Location }\end{array}$} & \multirow{2}{*}{$\begin{array}{l}\begin{array}{l}\text { Shut- } \\
\text { down } \\
\text { Period } \\
\text { (Days) }\end{array} \\
1\end{array}$} \\
\hline & & & $5 / 21 / 69$ & 7 & & & Row & 4 & \\
\hline & 35 & $5 / 29 / 69$ & $6 / 26 / 69$ & 28 & 1202 & 42.93 & $"$ & 4 & 8 \\
\hline & $\begin{array}{l}36 A- \\
38 A\end{array}$ & $6 / 30 / 69$ & $9 / 28 / 69$ & 75 & 3166 & 42.21 & out & & 19 \\
\hline & $38 B$ & $10 / 3 / 69$ & $10 / 26 / 69$ & 13 & 600 & 46.15 & Row & 4 & 5 \\
\hline & $39 \AA$ & $12 / 16 / 69$ & $1 / 10 / 70$ & 25 & 771 & 30.84 & $"$ & 4 & 61 \\
\hline & $39 B$ & $1 / 14 / 70$ & $1 / 19 / 70$ & 5 & 185 & 37.00 & $"$ & 4 & 4 \\
\hline & $39 C$ & $1 / 19 / 70$ & $1 / 28 / 70$ & 9 & 415 & 46.11 & $"$ & 4 & 0 \\
\hline & $40 A$ & $1 / 31 / 70$ & $2 / 10 / 70$ & 10 & 443 & 44.3 & $"$ & 4 & 3 \\
\hline & $40 B$ & $2 / 10 / 70$ & $3 / 1 / 70$ & 19 & 924 & 48.63 & $"$ & 4 & 0 \\
\hline & $41 A$ & $3 / 5 / 70$ & $3 / 25 / 70$ & 20 & 961 & 48.05 & $"$ & 4 & 4 \\
\hline & $41 B$ & $3 / 27 / 70$ & $4 / 1 / 70$ & 5 & 157 & 31.40 & $"$ & 4 & 2 \\
\hline & $42 A$ & $4 / 18 / 70$ & $5 / 18 / 70$ & 30 & 1344 & 44.80 & $"$ & 4 & 17 \\
\hline & $43 A$ & $5 / 26 / 70$ & $6 / 23 / 70$ & 28 & 1350 & 48.21 & $"$ & 4 & 8 \\
\hline & $44 A$ & $6 / 26 / 70$ & $7 / 15 / 70$ & 19 & 900 & 47.37 & $"$ & 4 & 3 \\
\hline & $44 B$ & $7 / 25 / 70$ & $8,4 / 70$ & 10 & 451 & 45.10 & $"$ & 4 & 10 \\
\hline & $45 A$ & $8 / 9 / 70$ & $8 / 23 / 70$ & 14 & 645 & 46.07 & $"$ & 4 & 5 \\
\hline & $45 B$ & $8 / 26 / 70$ & $9 / 10 / 70$ & 15 & 705 & 47.00 & $"$ & 4 & - \\
\hline
\end{tabular}


32

1
1
1 


\section{APPENDIX B \\ PROCEDURE FOR THE DISSOLUTION OF EBR-I! IRRADIATED \\ NICKEL CAPSULES CONTAINING $\mathrm{PuO}_{2}$}

\section{Acid Dissolution}

1. Clean outside of nickel capsule by immersing it in $6 \underline{\mathbf{N}} \mathrm{HNO}_{3}$ until a fresh nickel surface is exposed.

2. Rinse nickel capsule with distilled water.

3. Place nickel capsule in a 250-ill round bottom quartz flask which has an outer $24 / 40 \$$ joint.

4. Place quartz flask in a heating mantel which is regulated by a Variac.

5. Connect a quartz water-cooled condenser. The jacket length is about $20 \mathrm{~cm}$. The condenser has an inner $24 / 40 \$$ joint for connection to the flask.

6. Add $50 \mathrm{ml} 9 \mathrm{~N} \mathrm{HBr}-0.1 \mathrm{NHF}$ which contains $10 \mathrm{mg}$ chloroplatinic acid (Note 1 j to the quartz flask.

7. Turn on the heating mantel and adjust the Variac setting to 80 .

8. Heat the solution while under reflux until the dissolution of the nickel capsule is complete (3-5 hours).

9. Turn off the heating mantel and allow the solution in the quartz flask to cool to room temperature.

10. Add $10 \mathrm{ml} 9 \mathrm{~N} H B r-0.1 \mathrm{~N}$ HF which contains $100-\mu 1$ of bromine to the quartz flask.

11. Turn on the heating mantel and adjust the Variac setting to 80 .

12. Heat the solution while under reflux for 2 hours.

13. Turn off the heating mante? and allow the solution in the quartz flask to cool to room temperature.

14. Place the quartz flask in an ice bath. 
15. Pass hydrogen bromide gas into the solution at a rate of approximately 3-4 bubbles per second via a $0.5 \mathrm{~mm}$ i.d. quartz tube which is long enough to pass through the condenser and into the solution (Note 2).

16. Place the quartz flask back in the heating mantel.

17. Raise the quartz tube out of the solution in the flask.

18. Wash the quartz tube with $10 \mathrm{ml} 12 \mathrm{~N} \mathrm{HBr}-0.5 \mathrm{~N} \mathrm{HF}$ (Note 3) and collect the washings in the quartz flask.

19. Remove the quartz tube from the condenser.

20. Turn on the heating mantel and adjust the Variac setting to 80 .

21. Heat the solution while under reflux for 8 hours.

22. Turn off the heating mantel and allow the solution to cool to room temperature.

23. Transfer approximately half the solution to a 50-ml quartz centrifuge tube.

26. Centrifuge for 15 minutes.

25. Transfer the supernate to a pre-weighed (Note 4) quartz Erlenmeyer flask.

26. Transfer the remaining solution in the round bottom quartz flask to the $5 \mathrm{C}^{-} \mathrm{ml}$ quartz centrifuge tube.

27. Centrifuge for 15 minutes.

28. Transfer the supernate to the pre-weighed quartz Erlenmeyer flask.

29. Wash the round bottom quartz flask with $15-20 \mathrm{ml}$ of distilled water and trunsfer this washing to the quartz centrifuge tube.

30. Wash the round bottom quartz flask with another 15-20 ml of distilled water and transfer this washing to the quartz centrifuge tube.

31. Centrifuge for 15 minutes.

32. Transfer the supernate to the pre-weighed quartz Erlenmeyer flask.

33. Wash the precipitate in the quartz centrifuge tube with $10 \mathrm{ml}$ distilled water.

34. Centrifuge for 15 minutes. 
35. Transfer the supernate to the quartz Erlenmeyer flask.

36. Carefully add $10 \mathrm{ml}$ aqua regia (4 parts $\mathrm{HCl}+1$ part $\mathrm{HNO}_{3}$ ) to the residue in the quartz certrifuge tube.

37. Place the guartz centrifuge tube in a hot water bath $\left(80-90^{\circ} \mathrm{C}\right)$ and heat 4 to 8 hours.

38. Carefully add $9 \mathrm{~N} \mathrm{HBr}$ dropwise to the solution in the quartz centrifuge tube until all the nitrate present is destroyed. (Note 4)

39. Remove the quartz centrifuge tube from the hot water bath and allow the solution to cool to room temperature.

40. Centrifuge for 15 minutes.

41. Transfer supernate to the quartz Erlenmeyer flask.

42. Wash the precipitate in the quartz centrifuge tube with $10 \mathrm{ml}$ distilled water.

43. Ceritrifuge for 15 minutes.

44. Transfer supernate to the quartz Erlenmeyer flask.

45. Repeat steps 42,43 , and 44 .

\section{Pyrosulfate Fusion}

1. To the residue in the quartz centrifuge tube, add $0.5 \mathrm{ml}$ conc. $\mathrm{H}_{2} \mathrm{SO}_{4}$.

2. Place cuartz tube halfway dowil into a tube furnace and heat to fumes of $\mathrm{H}_{2} \mathrm{SO}_{4}$. (Note 5)

3. Remove quartz tube from tube furnace and allow to cool to room temperature.

4. Add 1 gram anhydrous $\mathrm{Na}_{2} \mathrm{SO}_{4}$ to the quartz tube.

5. Place quartz tube halfway down into the tube furnace and adjust heat until the $\mathrm{NaHSO}_{4}$ formed by the reaction of $\mathrm{Na}_{2} \mathrm{SO}_{4}$ has melted.

6. Heat the sample for 12 hours while applying enough heat to keep the salt molten.

7. Occasionally add 1-2 drops of conc. $\mathrm{H}_{2} \mathrm{SO}_{4}$ to the quartz tube. (Note 6) 
8. Remove the quartz tube from the furnace and allow it to cool to room temperature.

9. Add $10 \mathrm{ml} ! \underline{\mathrm{N}} \mathrm{HNO}_{3}$ to the quartz tube.

10. Place the quartz tube in a hot water bath at $80-90^{\circ} \mathrm{C}$ and heat for several hours until the fusion melt dissolves.

11. Centrifuge for 15 minutes.

12. Remove a 10-ul aliquot of the solution in the centrifuge tube and test for the presence of plutonium by hexone extraction and alpha counting. (Note 7)

13. Transfer the residue which remains from the $\mathrm{NaHSO}_{4}$ fusion to a piece of filter paper.

14. Count residue with a portable alpha survey meter such as an Eberline Model PAC-4G. (Note 8)

Notes

1. The presence of $10 \mathrm{mg}$ of chloroplatinic acid increases the dis-

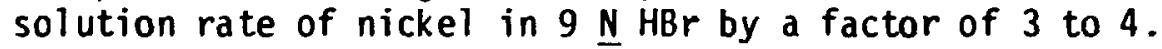

2. The $\mathrm{HBr}$ concentration of the solution will be increased to approximately $12 \mathrm{~N}$. Under these conditions, $\mathrm{NiBr}_{2}$ (brown in color) will precipitate. However, as the solution is heated in step 21 and the $\mathrm{HBr}$ concentration decreases, the precipicate will go into solution.

3. Prepare $12 \underline{\mathrm{N}} \mathrm{HBr}$ by passing $\mathrm{HBr}$ gas into a solution of $9 \underline{\mathrm{N}} \mathrm{HBr}$ in ar. ice bath.

4. Assume all the nitrate is destroyed when further additions of $9 \mathrm{~N}$ $\mathrm{HBr}$ do not cause bromine evolution.

5. Fuming with $\mathrm{H}_{2} \mathrm{SO}_{4}$ insures that all $\mathrm{H}_{2} \mathrm{O}$ has been driven off.

6. The excess $\mathrm{H}_{2} \mathrm{SO}_{4}$ present from the occasional dropwise addition of $\mathrm{H}_{2} \mathrm{SO}_{4}$ will reflux and wash down the sides of the quartz tube.

7. Less than $0.001 \%$ of the plutonium in the samples was found in the solution resulting from the $\mathrm{NaHSO}_{4}$ fusion.

8. When little or no alpha activity can be detected in the residue from the dissolution, discard the residue. If significant alpha activity remains, repeat the fusion. 